\title{
Cell landscape of cerebrospinal fluid and neuroinflammatory signatures in the bacterial meningitis through high-throughput sequencing and meta-analysis
}

\section{Tingyi Wen ( $\nabla$ wenty@im.ac.cn )}

Institute of Microbiology, Chinese Academy of Sciences

Haihan Xiao

Institute of Microbiology, Chinese Academy of Sciences

Haijuan Xiao

Beijing Children's Hospital, Capital Medical University

\section{Yun Zhang}

Institute of Microbiology, Chinese Academy of Sciences

Lingyun Guo

Beijing Children's Hospital, Capital Medical University

\section{Zhenzhen Dou}

Beijing Children's Hospital, Capital Medical University

\section{Linlin Liu}

Beijing Children's Hospital, Capital Medical University

\section{Liang Zhu}

Beijing Children's Hospital, Capital Medical University

\section{Wenya Feng}

Beijing Children's Hospital, Capital Medical University

\section{Bing Liu}

Beijing Children's Hospital, Capital Medical University

Bing Hu

Beijing Children's Hospital, Capital Medical University

Tianming Chen

Beijing Children's Hospital, Capital Medical University

Gang Liu

Beijing Children's Hospital, Capital Medical University

\section{Research Article}


Keywords:

Posted Date: January 27th, 2022

DOI: https://doi.org/10.21203/rs.3.rs-1290782/v1

License: (c) (1) This work is licensed under a Creative Commons Attribution 4.0 International License. Read Full License 


\section{Abstract}

\section{Background}

Bacterial meningitis (BM) is a life-threatening infection occurring in the central nervous system (CNS). Current understandings about cell participations in neuroinflammation of BM are obscure.

\section{Methods}

Cerebrospinal fluid (CSF) and blood samples were extensively collected from BM patients in different onset stages. High-throughput sequencing was conducted to decipher the expression profiles of CSF cells and peripheral blood leukocytes (PBLs) in single-cell and bulk-cell levels, and meta-analysis was carried out to comprehensively analyze the characteristics of CSF cells in BM progression in combination with the clinical features of patients.

Results

Implementation of single-cell RNA-sequencing (SCRNA-SEQ) identified 18 immune cell subsets in CSF and characterized their population structure and dynamics in BM progression. Two novel neutrophil (NEU) and monocyte (MO) subtypes, cytokine ${ }^{+} \mathrm{NEU}$ and cytokine ${ }^{+} \mathrm{MO}$, were discovered, which contributed to the increase of cytokine levels in CSF supernatant and played pioneering roles against bacterial invasion. The CSF of BM patients with unsatisfactory therapeutic effects presented different cell heterogeneities compared with those with satisfactory therapeutic effects, which featured changed intercellular communications and increased proportions of myeloid type- $₫$ dendritic cells (mDC2s) and plasmacytoid dendritic cells (pDCs). In sepsis-developed BM, CSF cells and PBLs exhibited distinct molecular hallmarks against CNS infection and sepsis, and CSF always contained a higher proportion of macrophages (MФs) and almost no platelets than PBLs in the entire onset period.

\section{Conclusion}

Our study conducted in-depth explorations about the characteristics of CSF cells in BM progression, which provided novel insights about immune cell engagements in acute CNS infection.

\section{Background}

$\mathrm{BM}$ is a common infectious disease occurring in the CNS [1]. Its incidence remains relatively high in preterm infants or neonates with documented sepsis [2,3]. Up to half of survivors have poor prognosis, who develop seizures, cognitive deficiencies or hearing and visual impairments [4]. In addition, although $\mathrm{BM}$ is considered an acute infection and could be treated favorably with timely antimicrobial therapy and clinical care [5-8], there have been an increasing number of BM cases facing the challenges of refractory conditions $[9,10]$, which always experience unsatisfactory therapeutic effects, long onset durations and illness relapse. All these indicate that BM is a significant threat to the health of children and needs more concern. 
CSF is a liquid mainly secreted by the choroid plexus and is contained in the ventricles of the brain and in the cranial and subarachnoid spaces [11]. As a physiological part of the CNS, CSF is not only an important material in the diagnosis of BM condition but also the cells provide insights to understand the cellular framework engaging in neuroinflammation of CNS in BM progression since nerve tissue is almost inaccessible [12]. Although it has been found that the occurrence of BM is always accompanied by pleocytosis and elevated polymorphonuclear (PMN) cells in CSF [13], the biological characteristics of CSF cells have not been comprehensively elucidated. Unlike extensive research on the biochemistry of CSF supernatant, such as glucose, protein and cytokines [14-17], there are no studies that dissect the characteristics of CSF cells in acute CNS infectious diseases. In general, knowledge about CSF cells is at a preliminary level, which leads to the current understandings about the participation of immune cells in CNS during BM progression are impercipient.

Herein, we analyzed the cellular information of CSF samples from the reports of their routine examinations during clinical treatment, to clarify the relationship between CSF cell characteristics and BM onset conditions. SCRNA-SEQ [18] was performed to characterize the cell landscape of CSF in different BM onset stages, aiming to identify cell subtypes, population structure and its changing law in the development of BM. Bulk RNA sequencing (bulkRNA-SEQ) was carried out to profile the transcriptomes of autologous PBLs and CSF cells from BM patients who developed by sepsis, hoping to find their different molecular hallmarks between in sepsis and CNS infection. Differences between CSF cells of BM patients with satisfactory and unsatisfactory therapeutic effects were also studied. Through performing in-depth explorations of the characteristics of CSF cells, we hope our work could provide new insights for understanding the engagements of immune cells in the CNS during BM progression.

\section{Methods}

\section{Sample processing}

The inclusion criteria of BM patients were in accordance with those described in related study, and all the patients received appropriate treatment [19]. BM cases caused by congenital (e.g., inner ear malformations, dermal sinus tracts) or acquired (e.g., head injuries, basal skull fractures) anatomical defects were excluded. BM patients with primary (e.g., antibody deficiency) or secondary immunodeficiency (e.g., immunosuppressive therapy) were also excluded. Fresh CSF and blood were obtained via lumbar and vein puncture, respectively. CSF cells and supernatant were separated and collected by direct centrifugation with $1800 \times$ rpm. Blood sera were collected by centrifugation with 2000xrpm, and blood leucocytes were collected after being treated with ammonium-chloride-potassium

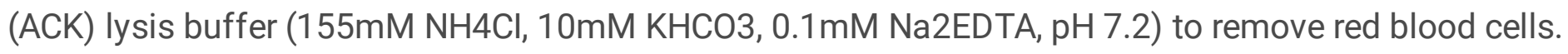
The sampling processes were under approval of the Ethics Committee of Beijing Children's Hospital, Capital Medical University, and performed according to the Declaration of Helsinki. Written informed consents were obtained from families prior to analysis of CSF samples and data. Detailed information of BM patients, as well as CSF and peripheral blood samples and their applied tests, was shown in Table. S1. 
293T cells were cultured in dulbecco's modified eagle medium (DMEM) supplemented with $10 \%$ fetal bovine serum (FBS, Gibco), 2mM L-glutamine (Gibco) and 100units/ml penicillin-streptomycin (Thermo) under $37^{\circ} \mathrm{C}$ and $5 \% \mathrm{CO} 2$ condition, and collected by centrifugation with $1800 \times$ rpm.

\section{Library preparation for SCRNA-SEQ and bulkRNA-SEQ}

A mimic assay based on QUARTZ-SEQ2 [20] was adopted here for library preparations for SCRNA-SEQ and bulkRNA-SEQ. For SCRNA-SEQ, CSF cells, PBLs and 293T cells were stained with $2 \mu \mathrm{M}$ calcein-AM (MCE) for 30 minutes on ice and were resolved in phosphate buffered solution (PBS) supplemented with $0.5 \%$ fetal bovine serum (FBS, Gibco) for the next step directly or RNAlater solution (Invitrogen) if unhurried. Stained cells were passed through a $40 \mu \mathrm{m}$ filter (Aladdin) and analyzed using MoFol XDP (Beckman Coulter). Single cells with positive green fluorescence were sorted into two 384-well plates, which contained the primer set eMDRT0001 - eMDRT0768 for cell barcoding. Reverse transcription and whole-transcript amplification were carried out in accordance with the protocols of QUARTZ-SEQ2.

For bulkRNA-SEQ, the RNAprep Pure Cell Kit (DP430, TIANGEN) or RNAprep Pure Micro Kit (DP420, TIANGEN) was used to extract cellular total RNA of CSF cells and PBLs with cell counts over or below $100 \times 10^{6} / \mathrm{mL}$, respectively, according to the manual. Fifty nanograms of total RNA was reverse transcribed into complementary DNA (CDNA) with primer X910 and then amplified under the same reaction conditions as described in SCRNA-SEQ.

The preparation processes of sequencing libraries for scRNA-SEQ and bulkRNA-SEQ were the same. Briefly, 100ng of amplified 2nd-strand cDNA was incubated for 5 minutes at $32^{\circ} \mathrm{C}$ and smeared into $400 \mathrm{bp}$ fragments by fragmentation module (NG305, TIANGEN). After purification, fragmented cDNA was end-repaired (NG302, TIANGEN) and ligated (NG303, TIANGEN) with $0.35 \mu \mathrm{M}$ truncated sequence adaptors (rYshapeP5-rYshapeP7LT series in QUARTZ-SEQ2). The library was further amplified and purified under the conditions described in QUARTZ-SEQ2.

\section{Library sequencing of scRNA-SEQ and bulkRNA-SEQ}

Library DNA was sequenced using HiSeq X Ten (Illumina). Sequencing primers for Read1 and Read2 were the same as described in QUARTZ-SEQ2, and the Index 1 sequencing primer was replaced by X908 for sample index. Sequence specification was as follows: Read1, 151 cycles; Index 1, 8 cycles; Read2, 151 cycles. The pooled raw data from one lane were demultiplexed into FASTQ files of each sample according to the index sequence in the cellranger software package (10X genomics). Clean data were generated after the reads with polluted adapters, Ns and low-quality reads were filtered out in R with Fastp package [21]. Extraction of cell barcodes (768 for scRNA-SEQ data and 1 for bulkRNA-SEQ data) and unique molecular identifiers (UMIs), reads mapping to human genome GRCh38.p13 and generation of cell-count matrix were processed in the combined workflow of UMI-tools, STAR, Subread and Samtools. The primer sequences of X910 and X908 are shown in Table. S2. 


\section{Data analysis of scRNA-SEQ and bulkRNA-SEQ}

Secondary analysis of SCRNA-SEQ and bulkRNA-SEQ was mainly carried out in the R environment. scRNA-SEQ data were analyzed in the Seurat package, including data normalization, feature selection of genes with high variability, linear dimension reduction, t-distributed stochastic neighbor embedding (tSNE) clustering and feature gene identification. Harmony [22] was used in integrative clustering for multiple scRNA-SEQ data. For cell type annotation, general cell types were annotated in the SingleR package through comparison with the HumanPrimaryCellAtlasData database from the Celldex package, and were further specified and confirmed with related knowledge from published papers according to the identified featured genes of cell clusters. Differentially expressed genes (DEGs) in transcriptomic comparison were analyzed in the DESeq2 package. Gene ontology (GO) enrichments were analyzed in the ClusterProfiler package. Cell trajectory and pseudotime analyses were carried out in the Monocle2 package.

Cell-cell communication analysis was performed in CellPhonedb in Python environment. Ligand-receptor interactions expressed in more than $10 \%$ of the cells in the corresponding subclusters were considered. Interactions with $P$ values $<0.05$ between two cell types were considered as truly connection.

\section{Reverse transcription and quantitative real-time polymerase chain reaction ( $R T-q P C R)$}

Total RNA of $1 \times 10^{5}$ CSF cells and PBLs was extracted, respectively, using an RNAprep pure Micro Kit (DP420, TIANGEN) according to the manufacturer's recommended procedure. First-strand cDNA was generated from 50ng total RNA using a FastKing RT Kit (KR116, TIANGEN). Real-time PCR was performed using SYBR green on the Roche LightCyler 480 system. The final $20 \mu l$ reaction mixture contained 300nM each primer, $1 \mu \mathrm{l}$ of cDNA, and $10 \mu \mathrm{l}$ of GoTaq qPCR Master Mix (Promega). All reactions were run in triplicate. Real-time PCR was initiated with a 3-min hot-start denaturation step at $95^{\circ} \mathrm{C}$ and then performed for 40 cycles at $95^{\circ} \mathrm{C}$ for $15 \mathrm{~s}$ and $60^{\circ} \mathrm{C}$ for 1 minute. $\beta$-actin was employed as reference genes. The relative expression level of interest gene was determined by comparing its cycle threshold $\left(\mathrm{C}_{\mathrm{T}}\right)$ value to the reference genes using the formula $2^{-\triangle C T}$. Primer sequences of TREM2, SLCO2B1 and $\beta$-actin are listed in Table. S3.

\section{Cell immunostaining and flow cytometry (FCM)}

All operations in this section were performed at low temperature. CSF cells and blood leucocytes were blocked for 15 minutes in PBS containing $0.5 \%$ bovine serum albumin (BSA, Sigma-Aldrich, A1933) and

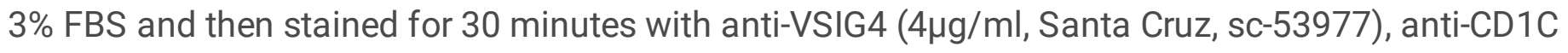
$(2 \mu \mathrm{g} / \mathrm{ml}$, NOVUS, NBP2-62220) or equal concentrations of isotype-matched control antibodies. After being washed three times with PBS containing 3\% FBS, the suspension was secondarily stained with 
$2 \mu \mathrm{g} / \mathrm{ml}$ Alexafluor 488-conjugated goat anti-mouse antibody (4408S, Cell Signaling) for 30 minutes in PBS containing $0.5 \%$ BSA and $10 \%$ goat serum (B900780, Proteintech) and then stained for another 5 minutes with $1 \mu \mathrm{g} / \mathrm{ml}$ Hoechst 33342. Cells were washed three times and fixed in PBS containing $1 \%$ paraformaldehyde. Cellular fluorescence was measured using an Influx flow cytometer (BD), and data were analyzed in FlowJo software.

\section{Enzyme-linked immunosorbent assay (ELISA) of cytokines}

CSF supernatants were obtained by direct CSF centrifugation with 5 minutes at $1500 \mathrm{rpm}$ under $4{ }^{\circ} \mathrm{C}$. Fresh blood was treated with anticoagulant, and serum was obtained by centrifugation for 10 minutes at $2000 \mathrm{rpm}$ at $4^{\circ} \mathrm{C}$. Interleukin-8 (CXCL8), pigment epithelium-derived factor (PEDF) and osteopontin (OPN) were measured by commercially available sandwich-type ELISA (R\&D Systems) following the manufacturers' instructions.

\section{Statistical analysis}

Statistical analysis was performed in GraphPad Prism. t-test was used to evaluate significant differences between two datasets. Chi-square tests were used to evaluate the contingency of cases. A significant effect was indicated by a $P$ value $<0.05$.

\section{Results}

\section{Study cohort}

Fifty-four BM patients were chosen for the study from the Department of Infectious Diseases of Beijing Children's Hospital. Their onset age ranged from 1 day to 2490 days, with a median of 68.5 days (Fig. 1A). Nineteen patients were infected by Streptococcus agalactiae(GBS), 11 patients were Streptococcus pneumonia, 10 patients were Escherichia coli, 2 patients were Listeria monocytogenes, and 2 patients were infected by Enterococcus faecium and Neisseria meningitidis. The remaining 6 patients were diagnosed by typical symptoms and CSF examinations without identified pathogens (Fig. 1B). Total 141 CSF and 39 peripheral blood samples were collected from BM patients during their clinical treatment (Fig. 1C).

\section{Classifying CSF samples and their represented BM onset conditions}

Cytological examination of CSF is important for the diagnosis of BM [14]. Normal CSF had cells in the range of $0 \sim 15 \times 10^{6} / \mathrm{ml}$. BM occurrence accompanied with pleocytosis of CSF, in which CSF cells were in abnormal condition with increased total cell counts (TCCs) above the normal range. BM patients with 
abnormal CSF cells were considered as in illness condition. Based on the above knowledge, we classified the 141 CSF samples and their represented BM onset conditions, through combined analysis of cellular characteristics of CSF with clinical characteristics of BM patients, to better tap their scientific values in other tests of this study.

According to the BM onset duration when CSF sampling and whether the number of CSF cells was in normal state or not, the $141 \mathrm{CSF}$ samples were found that representing three stages of BM (Fig. 2A): initial onset (onset duration within the first four days after BM occurrence and abnormal CSF cells), remission (onset duration above four days and abnormal CSF cells) and recovery (normal CSF cells). The remission stage was further divided into unrefractory and refractory remission, in which unrefractory remission meant that BM patients acquired satisfactory therapeutic effects with a relatively short remission time, while refractory remission meant that BM patients suffered unsatisfactory therapeutic effects with a significantly longer remission time.

The 141 CSF samples were further specified into nine groups, which are represented by S1 S9 (Fig. 2B). The TCCs and proportions of PMN and mononuclear (MN) cells of CSF samples in each group were showed out. S1 represented the BM initial onset stage, in which CSF had higher TCCs (average $2764 \times 10^{6} / \mathrm{ml}$ ) and higher proportion of PMN cells (average 78\%) than other groups. CSF samples in the unrefractory remission stage were divided into group S2 and S3, as they presented distinct CSF cell characteristics, in which the proportion of PMN cells in cells of each S2-CSF sample was over $50 \%$, while to S3-CSF samples, the value was below $50 \%$. CSF samples of S2 also had higher TCCs than those in group S3. CSF samples in S4 and S5 represented the recovery stage after unrefractory remission. In S4, TCCs were in the normal range and PMN cells almost disappeared. In S4, TCCs further declined and were marked as zero in the reports of clinical routine examination as it was lower under the detection limit. CSF samples in the BM refractory remission stage were divided into groups S6 and S7, which had similar cell characteristics corresponding to S2 and S3, respectively. S8 and S9 represented the recovery stage after refractory remission, which were similar to $\mathrm{S} 4$ and $\mathrm{S} 5$, respectively.

Taking PBLs as a reference, the representative characteristics of CSF cells in groups S1-S9 were checked and confirmed through FCM (Fig. 2C). CSF had similar basic cell groups like PBLs, which were composed of PMN cells and $\mathrm{MN}$ cells. PMN cells and $\mathrm{MN}$ cells were indeed changed in the development of BM, which was in accordance with the reports of routine clinical CSF examinations. Noticeably, CSF in S3 and S8 actually contained a few PMN cells, although it was marked as zero in the routine examination report. Similarly, CSF in S4 and S9 was also detected with the existence of few cells.

\section{Cell landscape of CSF in BM patients by SCRNA-SEQ}

Through CSF cell separation, living single-cell sorting by FCM, scRNA-SEQ and analysis, we obtained single-cell transcriptomes of 15960 CSF cells from the S1 (2524 cells), S3 (2896 cells), S4 (2246 cells), S6 (1930 cells), S7 (3854 cells) and S8 (2537 cells) groups, which covered the main onset conditions and key time points in BM development (Fig. 3A). We first analyzed the scRNA-SEQ data of CSF cells in each 
group independently. Multiple published papers were referred for cell annotation [23-30] with the feature genes of clustered cell subsets. Fourteen immune cell subtypes, including NEUs, MOs, MФs, mDCs, T cells, pDCs, B cells and plasma cells, were identified with uneven distribution in the CSF with different BM onset conditions. Certain amounts of apoptotic cells were also identified featuring apoptosis-related genes such as TMBIM4 [31], RNASE1 [32] and KAT7 [33]. This result showed the basic cell subtypes and cellular population structure of CSF in different BM stages. In general, the results showed that decreased TCCs of CSF were accompanied by a decreased proportion of PMN cells (NEUs) and an increased proportion of MN cells ( $T$ cells, MOs, MФs, mDCs, pDCs, B cells and plasma cells) in BM progression (Fig. 3B), which was in accordance with the routine CSF examination data. Meanwhile, they also revealed that decreased TCCs of CSF were accompanied by a decreased proportion of myeloid cells (NEUs, MOs, MФs and $\mathrm{mDCs}$ ) and an increased proportion of lymphoid cells (T cells, B cells, pDCs and plasma cells) in BM progression (Fig. 3C).

To verify the accuracy of scRNA-SEQ data, extra scRNA-SEQ of 293T cells was carried out in parallel. CSF cells and 293T cells were clearly distinguished from each other after mixed clustering (Fig. S1). Incidentally, we also obtained single-cell transcriptomes of 424 CSF cells in group S5 from sample C138 (Fig. S2). These cells were clustered as 228 naïve T cells, 153 effector T cells, 24 myeloid cells and 19 B cells. The proportion of lymphoid cells (94.3\%) further verified the above conclusion: lower TCCs of CSF and a higher proportion of lymphoid cells within it. Unfortunately, as the age of the donor patients was old (6 years old), we carefully did not include this result in the official data.

To specify the cell types of CSF in BM, we extracted myeloid cells and lymphoid cells in each stage and integratively subclustered them. Nine myeloid cell subtypes and nine lymphoid cell subtypes (Fig. 3D) and their featured genes (Fig. 3E) were identified. In other words, we officially characterized 18 cell subtypes within CSF in BM. The 18 CSF cell subtypes included 2 NEU subtypes: conventional NEUs (cNEUs) and cytokine ${ }^{+}$NEUs, which commonly expressed FCGR3B, CSF3R, SLC25A37 and S100A8/A9, while cytokine ${ }^{+}$NEUs also highly expressed FFAR2, IL1RN, TNFAIP6 and GOS2. Two MO subtypes, conventional MOs (cMOs) and cytokine ${ }^{+}$MOs, commonly featured S100A8/A9, VCAN, FCN1 and CD36 expression, while cytokine ${ }^{+}$MOs featured THBS1, SLC39A8, CCL2/4/20 and CCL3L 1 expression. One MФ cell type expressed $C 1 Q A / B / C, L Y V E 1, A P O E, P L T P, D A B 2, F O L R 2$ and GPNMB. Four mDC subtypes [34], mDC2s, SIGLEC6 ${ }^{+} \mathrm{AXL}^{+} \mathrm{mDCs}$, type- $\triangle \mathrm{mDCs}$ (mDC1s) and $\mathrm{CCR} 7^{+} \mathrm{mDCs}$, commonly had high expression of $H L A-D P A 1 / B 1$ and $H L A-D Q A 1 / A 2 / B 1$. mDC2s specifically had high expression of FCER1A, CD1C and CLEC1OA; SIGLEC6 ${ }^{+} \mathrm{AXL}^{+} \mathrm{mDCs}$ specifically had high expression of SIGLEC6, TCF4, $B C L 11 A, A X L$ and UGCG; mDC1s highly expressed XCR1, CLNK, CLEC9A, C1orf54, DNASE1L3, CADM1, CPVL, IRF8 and WDFY4; and CCR7 ${ }^{+} \mathrm{mDC}$ s highly expressed $L A M P 3, C C R 7, B I R C 3, C D 83$, MARCKSL 1 , CCL22, FSCN1 and IDO1. Four a $\beta$ T subtypes, naïve T cells, $C D 8^{+} \mathrm{T}$ cells, CD4 ${ }^{+} \mathrm{T}$ cells and exhausted $\mathrm{T}$ cells, commonly had high expression of TRAC, TRBC1 and TRBC2. Naïve T cells featured IL7R, TCF7, LTB and $L E F 7 ; C D 8^{+} T$ cells featured $C D 8 A / B$ and $G Z M H ; C D 4^{+} T$ cells featured $C D 4, M A F, M I A T$ and

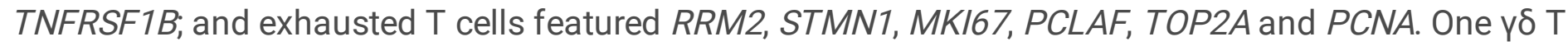
cell type featured TRGC1, KLRG1 and ZBTB16 expression. One natural killer (NK) cell type featured GNLY, 
KLRD1 and CTSW expression. One B cell type featured MS4A1, CD79A/B, IGHD, BANK1 and BIRC3 expression. One pDC cell type featured TCF4, IRF8, UGCG, MPEG1, BCL11A, SERPINF1, TSPAN13 and $G Z M B$ expression. One plasma cell type featured $M Z B 1, X B P 1, I G H G 1 / 3 / 4, I G L C 2$ and IGKC expression. Among these cell types, cytokine ${ }^{+}$MOs and cytokine ${ }^{+}$NEUs shared the expression of FAM177B, TNFAIP3, CXCL8 and IL 1B. MФs and CMOs shared TMEM176A and TMEM176B expression. MФs, CMOs and cytokine ${ }^{+}$MOs shared CD163 expression. GZMA, NKG7 and CCL 5 were universally expressed on CD $8^{+} T$ cells, exhausted T cells, $\gamma \delta$ T cells and NKs. $\gamma \delta T$ cells and NKs shared the expression of TRDC and KLRB1. Plasma cells and B cells shared the expression of IGHM. Plsma cells and pDCs shared JCHAIN expression. CD4 was also highly expressed on pDCs. In addition, we also combined myeloid cells and lymphoid cells to perform integrated clustering (Fig. S3), which showed the general cell types in CSF: NEUs, MOs, MФs, mDCs (mainly mDC2s), naïve T cells, effector T cells, exhausted T cells, NKs, B cells, pDCs and plasma cells.

\section{The structure of the CSF cell population and its changes in BM progression}

After characterizing the cell types of CSF in BM, we further analyzed the structures of CSF cell populations in different BM stages, to reveal the changing law of CSF cell population in BM progression.

We first analyzed the structures of PMN cell groups (Fig. 4A) and MN cell groups (Fig. 4B) in CSF, at different stages of BM. The eighteen immune cells, which were identified by scRNA-SEQ, were demultiplexed into their original BM stages (Fig. S4), and the constitutions of PMN cells and MN cells in each BM stage were calculated. To PMN cell group, cNEUs and cytokine ${ }^{+}$NEUs existed in CSF with equal proportions in the initial stage of BM, and the proportion of cytokine ${ }^{+}$NEUs continued to decrease in the remission stage to the recovery stage. In the case of similar TCCs, PMN cells had fewer cytokine ${ }^{+}$NEUs with the extension of onset durations, such as in the comparison between CSF cells in S3 and S6 or in S4 and S8. Even if the CSF in S7 had higher TCCs than that in S8, the proportion of cytokine ${ }^{+}$NEUs did not increase. To MN cell group, it contained high proportions of cMOs and cytokine ${ }^{+} \mathrm{MOs}$ and low proportions of lymphoid cells in the initial onset stage of BM. The proportions of lymphoid cells increased a lot in the CSF at the unrefractory remission stage. T cells were dominated in the normal CSF. Although lymphoid cells still occupied an advantage in the CSF of the refractory remission stage, mDCs and pDCs were also in high proportions. Among all BM stages, CSF had stable proportions of MФs and B cells, and plasma cells were relatively more abundant in the CSF of S3 compared with those in other stages.

The populational structure of whole CSF cells (Fig. 4C) and quantitative characteristics of each cell type (Fig. 4D) in BM at different stages were then analyzed, which displayed the process that how the population structure of CSF cells changed in the real physiological process of BM. With further calculation (Fig. 4E), we could see that, in the BM period from initial onset to remission, the number of cNEUs, cytokine ${ }^{+}$NEUs, cMOs and cytokine ${ }^{+}$MOs declined obviously. For BM patients with satisfactory 
therapeutic effects, the process from remission to recovery was accompanied by decreased numbers of all cell types with relatively equal degrees. In contrast, in the process from remission to recovery of BM patients with unsatisfactory therapeutic effects, cNEUs, cMOs, mDC2s, naïve T cells, $\gamma \delta T$ cells and pDCs declined more obviously than other cell types. We also analyzed the TCC decline of CSF in the refractory remission stage. The number of cNEUs decreased most obviously, and cytokine ${ }^{+} \mathrm{NEUs,}$, cMOs and mDC2s also decreased considerably, while the number of $C D 8^{+} T$ cells and $\gamma \delta T$ cells increased in this process, which negatively contributed to the TCC decline in CSF.

\section{Cytokine ${ }^{+}$NEUs and cytokine ${ }^{+}$MOs undertook pioneering roles against bacterial invasion and mainly contributed to the increase of cytokine levels in CSF supernatant in BM}

The characteristics of cytokine ${ }^{+}$NEUs and cytokine ${ }^{+}$MOs were further analyzed, as they are two novel cell types participating in CNS immunity that have not yet been reported.

We know that cytokine ${ }^{+}$NEUs and cytokine ${ }^{+}$MOs abundantly exist in the CSF of the BM initial onset stage from the above results. We then analyzed the content of cytokine ${ }^{+} \mathrm{NEUs}$ and cytokine ${ }^{+} \mathrm{MOs}$ within CSF cells in the BM unrefractory and refractory remission stages, and the results showed that, compared with CSF in the unrefractory remission stage, fewer cytokine ${ }^{+}$NEUs (Fig. 5A) and cytokine ${ }^{+}$MOs (Fig. 5B) could be identified by scRNA-SEQ in the refractory remission stage, even if CSF in group S6 had higher numbers of PMN cells and TCCs. This indicated that cytokine ${ }^{+}$NEUs and cytokine ${ }^{+}$MOs preferred to exist in the CSF with short onset durations and may undertook the main roles of anti-bacterial infection, since pathogens would be removed under antibiotic treatment with the extension of onset duration.

We conducted GO enrichments on biological process (BP) targeting the feature genes of cytokine ${ }^{+} \mathrm{NEUs}$, cytokine ${ }^{+}$MOs, cNEUs, cMOs and MФs, as they were predominant cell types in the CSF of the BM initial stage (Fig. 5C). The results showed that the feature genes of cytokine ${ }^{+}$NEUs and cytokine ${ }^{+}$MOs, such as IL 1B, CCL2, CXCL8 and TNFAIP3, had a special enrichment in the immune response to bacterial molecules compared with other cell types. In addition, the pseudotime of CSF cell subtypes in the BM initial stage was analyzed (Fig. 5D), and it was found that cytokine ${ }^{+}$NEUs were located on the root state of trajectory evolution, which meant that cytokine ${ }^{+}$NEUs appeared earlier in CSF than CNEUs at the beginning of BM.

According to the characteristics of cytokine ${ }^{+}$NEUs and cytokine ${ }^{+} \mathrm{MOs}$, it could be predicted that CSF supernatant in the BM initial onset stage had especially high cytokine levels rather than that in remission and recovery stages. CSF of BM patients in the BM unrefractory remission stage had higher cytokine levels than that in the BM refractory remission stage. CSF in group S6 should not have higher cytokine levels than that in group S3, although it had higher TCCs and PMN cell numbers. To verify the characteristics of cytokine ${ }^{+} \mathrm{NEUs}$ and cytokine ${ }^{+} \mathrm{MOs}$, we measured the CXCL8 levels of CSF 
supernatants in the initial onset, remission and recovery stages of BM (Fig. 5E) and found that CSF in the initial onset stage contained significantly higher CXCL8 levels than those in other stages. CSF samples in the unrefractory remission stage of group S3 contained higher CXCL8 levels than those in the refractory remission stage of group S7. CXCL8 content was not different in the CSF between groups S3 and S6, although CSF samples in S3 had lower TCCs. CSF samples of group S7 were in abnormal BM conditions, but their CXCL8 content was low, similar to normal CSF samples. In addition, CSF samples with extended onset durations (groups S6, S7 and S8) contained more cases in which the CXCL8 concentrations were below the lower detection limit than those with short onset durations (groups S3, S4 and S5) (Fig. 5F).

\section{CSF cells of BM patients with unsatisfactory therapeutic effects featured higher proportions of mDC2s and pDCs than those with satisfactory therapeutic effects}

The refractory condition of BM patient during clinical treatment should receive more attention. ScRNASEQ of CSF cells in unrefractory and refractory remission stages revealed different cell heterogeneities between them. We then performed further explorations about this difference.

The bulk transcriptomes of CSF cells in the unrefractory and refractory remission stages (Fig. 6A) were sequenced. DEGs were analyzed through transcriptomic comparison of CSF cells in the unrefractory remission stage versus those in the refractory remission stage. We found that the top down-regulated DEGs were mainly expressed on plasma cells (IGHA1, IGHG1/2, IGLV3-1, IL26, etc.) or cytokine ${ }^{+}$NEUs and cytokine ${ }^{+}$MOs (ANKRD22, GPR84, RETN, B4GALT5, etc.) (Fig. S5), while the top up-regulated DEGs were mainly expressed on mDC2s (CD1E, FCER1A, HLA-DPB1, HLA-DRB1, CD1C, etc.) and pDCs (LILRA4, CLEC4C, TSPAN13, TMEM8B, etc.) (Fig. S6). The top up-regulated DEGs had obviously lower adjusted $P$ (padj) values than the top down-regulated DEGs, which meant that the featured difference in population structure between CSF cells in the unrefractory and refractory remission stages was the difference of $\mathrm{mDC} 2 \mathrm{~s}$ and $\mathrm{pDCs}$. In other words, CSF cells in the unrefractory stage featured higher proportions of $\mathrm{mDC} 2 \mathrm{~s}$ and $\mathrm{pDCs}$ than those in the refractory remission stages.

We then measured CD1C expression on CSF cells through FCM-immunostaining and PEDF (encoded by SERPINF1) levels in CSF supernatants through ELISA in the unrefractory and refractory remission stages of BM. As the marker genes, CD1C expression and PEDF levels represented the contents of mDC2s and $\mathrm{pDCs}$ in the CSF, respectively. The results showed that CSF MN cells in the unrefractory remission stage contained higher proportions of $\mathrm{CD}_{1} \mathrm{C}^{+}$cells than those in the refractory remission stage (Fig. 6B). For PEDF, its levels in the supernatants of CSF samples in the unrefractory remission stage were significantly higher than those in the refractory remission stage (Fig. 6C).

\section{CSF cells of BM patients with unsatisfactory therapeutic effects had different intercellular communications}




\section{compared with those with satisfactory therapeutic effects}

After delineating the different population structures of CSF cells between the unrefractory and refractory remission stages, we then studied whether they had different cell-cell interactions as they were in different onset conditions. This analysis was performed in CSF samples from groups S3 and S7, as they had similar cell characteristics. scRNA-SEQ data of CSF cells in groups S3 and S7 were reanalyzed and integratively clustered to ensure that they had consistent cell types, and intercellular communications were analyzed in the cellphonedb package (Fig. 7A).

CSF cells in groups S3 and S7 were integratively clustered into 11 general cell types: NEUs, MOs, MФs, mDC1s, mDC2s, naïve T cells, effector T cells, exhausted T cells, B cells, pDCs and plasma cells. All cell types interacted with each other in both the unrefractory and refractory remission stages. Compared with those in the unrefractory remission stage, NEUs, MOs, MФs, naïve T cells, effector T cells and exhausted $T$ cells had decreased connections with other cell types, while mDC1s, mDC2s, B cells, pDCs and plasma cells had increased interactions in the refractory remission stage of BM (Fig. 7B).

Ligand-receptor interactions in each CSF cell type with changed levels between the unrefractory and refractory remission stages were then analyzed (Fig. 7C). The changed levels were represented by three types: weakened, enhanced and roved. Specifically, "weakened" and "enhanced" interactions meant that one cell type had this connection with fewer and more cell types in the refractory remission stage than in the unrefractory remission stage, respectively. "Roved" interaction meant that one cell type had this connection with different other cell types in refractory remission stages compared with those in the unrefractory remission stage. A total of 89 ligand-receptor interactions were identified with changed levels among all cell types (Table. S4). They included cytokine-receptor related interactions, cell-cell adhesion related interactions, immune response related interactions, immune suppression related interactions and immune checkpoint related interactions $[35,36]$. For example, LGALS9-related interactions were extensively active in the intercellular communications of MOs, MФs, mDC1s, mDC2s and B cells, but rarely in NEUs, T cells, and plasma cells.

Through further analysis, we found 11 and 4 ligand-receptor interactions that were down-regulated and up-regulated within CSF cells in the unrefractory remission stage compared with those in the refractory remission stage, respectively (Fig. 7D). "down-regulated" meant that the interactions occurred in fewer cell types in the refractory remission stage than in the unrefractory remission stage, while "up-regulated" meant the opposite performance. For example, the ANXA1_FPR2 interaction was attenuated among connections of NEUs with effector $\mathrm{T} /$ exhausted $\mathrm{T} /$ naïve $\mathrm{T} / \mathrm{MO} / \mathrm{mDC} 2$ cells and connections of $\mathrm{M \Phi s}$ with $\mathrm{MO} / \mathrm{NEU}$ cells in the refractory remission stage compared with that in the unrefractory remission stage. The AXL_GAS6 interaction was enhanced among connections of pDCs with $\mathrm{M} \Phi / \mathrm{mDC} 1 / \mathrm{mDC} 2$ cells in the refractory remission stage compared with that in the unrefractory remission stage. In addition, we also found 25 turned-off and 16 turned-on interactions that specifically occurred among CSF cells in the unrefractory and refractory stages, respectively (Fig. 7E). For example, the CD52_SIGLEC10 interaction was active in the connections between $\mathrm{T} / \mathrm{B}$ cells and $\mathrm{MO} / \mathrm{M} \Phi / \mathrm{mDC} 1$ cells in the unrefractory remission 
stage, but it was silenced among any cells in the refractory remission stage. The HGF_CD44 interaction was silenced in CSF cells in the unrefractory remission stage but was widely activated in the communication between plasma cells and other cell types in the unrefractory remission stage.

\section{Distinct molecular hallmarks of CSF cells and PBLs against CNS infection and sepsis in sepsis-developed BM}

Immunity in the CNS is considered an independent unit with special characteristics due to the presence of the blood brain barrier (BBB) and immune privilege $[37,38]$. We then explored whether CSF cells and PBLs exhibited different characteristics in sepsis-developed BM.

Multiple autologous CSF-blood pairs from septicemia-developed BM patients in different onset conditions were collected, and the characteristics in sepsis-developed BM progression were analyzed (Fig. $8 A$ ). We found that in the early stages of BM, the TCCs of most PBL samples were abnormal (normal range $4 \sim 10 \times 10^{9} / \mathrm{ml}$ ), which showed an onset condition of sepsis. If BM patients suffered refractory conditions, the TCCs of PBLs recovered to normal in the refractory remission stage of BM. This meant that when BM patients suffered refractory BM conditions with long remission durations, their sepsis symptoms were cured, which showed a recovered condition of sepsis. This phenomenon was confirmed with a significant difference (Fig. 8B).

Bulk transcriptomes of PBLs and CSF cells in different onset conditions of sepsis-developed BM were sequenced. Before comparing their transcriptomic differences, we first carried out SCRNA-SEQ of $351 \mathrm{PBL}$ cells (Fig. S7) and identified 7 cell types: NEUs (FCGR3B $\left.{ }^{+}, C S F 3 R^{+}, S L C 25 A 37^{+}\right)$, MOs $\left(C D 300 E^{+}, F C N 1^{+}\right.$, $\left.V C A N^{+}\right)$, naïve T cells $\left(T C F 7^{+}, I L 7 R^{+}, L E F 7^{+}\right)$, effector T cells $\left(C S T 7^{+}, N K G 7^{+}, G Z M A^{+}\right), \mathrm{B}$ cells $\left(V P R E B 3^{+}\right.$,

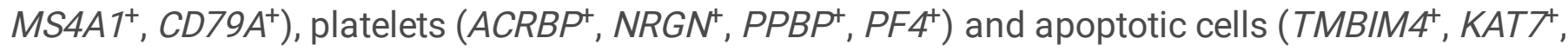
$R N A S E 1^{+}$). We also compared the bulk transcriptomes of PBLs in the onset stage versus the recovered stage and analyzed the DEGs (Fig. S8). We found that down-regulated DEGs had GO enrichments in NEUrelated immunity and immune responses to bacteria, while the up-regulated DEGs were enriched in $T$ cell activation and leukocyte cell-cell adhesion. The top down-regulated DEGs, such as $L I L R B 2, L I L R A 6, F G R$ and ETS2, were mainly expressed in MOs and NEUs, and the top up-regulated DEGs, such as GNLY, KLRG1, TRDC, GZMA and SH2D1A, were mainly expressed in effector T cells (Fig. S9). This revealed that the TCC increase in PBLs was accompanied by the growth of myeloid cells, especially NEUs, in sepsis occurrence, which was similar to CSF cells in BM.

According to the characteristics of sepsis-developed BM progression, we compared the bulk transcriptomic difference between pairwise CSF cells and PBLs in three groups: CSF cells in the initial onset stage of BM versus PBLs in the onset stage of sepsis (group 1), CSF cells in the unrefractory remission stage of BM versus PBLs in the onset stage of sepsis (group 2), and CSF cells in the refractory remission stage of BM versus PBLs in the recovered stage of sepsis (group 3) (Fig. 8C). Down-regulated DEGs in groups 1, 2 and 3 were marked as " $G 1^{-\prime \prime}$, "G2"” and "G3"”, respectively, while up-regulated DEGs in 
groups 1, 2 and 3 were marked as " $\mathrm{G} 1^{+"}$, " $\mathrm{G} 2^{+"}$ and " $\mathrm{G} 3^{+}$", respectively. Through analysis, we found that multiple G1- DEGs with top $P$ values, such as $C X C L 8, P I 3, S P P 1, C C L 20, C C L 2$, etc., were highly expressed in cytokine ${ }^{+}$NEUs, cytokine ${ }^{+}$MOs and MФs of CSF (Fig. S10), while multiple top $\mathrm{G}^{+}$DEGs were highly expressed in platelets (PPBP, PF4, SPARC, MYL9, etc.) and B cells (CD79A, IGKC, IGHM, etc.) of PBLs, or both of them ( $P D L I M 1)$ (Fig. S11). Multiple G2 DEGs with top $P$ values were mainly expressed in MФs (SPP1, APOE, C1QA, C1QC, GPNMB, etc.) and effector T/exhausted T cells (SCD, CCR5, CXCR6, FABP5, CTLA4, etc.) of CSF (Fig. S12), while multiple top $\mathrm{G}^{+}$DEGs were highly expressed in NEUs (S100P, S100A8, IFITM2, CYP4F3, etc.) and platelets (MYL9, NGRN, MPIG6B, TREML 1, etc.) of PBLs (Fig. S13). For G3- DEGs, multiple of them with high $P$ values were the feature genes of MФs and mDCs (CLEC10A, CD1E, HLA-DQA1, CLIC2, etc.), or both of them (MRC1, IL18, ATP1B1, GGTA1P, etc.) (Fig. S14). Meanwhile, the $\mathrm{G}^{+} \mathrm{DEG}$ with the top $P$ values included featured genes of platelets, naïve T cells ( $L R R N 3, M A L$, TCF7, ABLIM1, etc.), effector T cells (FGFBP2, ADGRG1, SPON2, etc.), B cells (IGHD, PAX5) and NEUs (CDA, S100A12, S100P, IFIT2/3, etc.) of PBLs (Fig. S15).

Further GO-BP enrichments showed that G1- DEGs were enriched in immune responses to bacteria and $\mathrm{G1}^{+}$DEGs were enriched in humoral immune response and blood coagulation. G2- DEGs were enriched in $T$ cell activation, cell chemotaxis, leukocyte proliferation and cell-cell adhesion, while $\mathrm{G}^{+}{ }^{+} \mathrm{DEG}$ s were enriched in NEU-related immunity, myeloid cell differentiation and blood coagulation. G3- DEGs had high enrichments in leukocyte proliferation, cell chemotaxis, cell-cell adhesion and antigen processing \& presentation, while $\mathrm{G}^{+}$DEGs were enriched in NEU-related immunity, mononuclear cell differentiation, humoral immune response and blood coagulation.

\section{CSF always contained a higher proportion of MФs and almost no platelets than PBLs in the entire onset period of sepsis-developed BM.}

From the above results, we could see that CSF cells always had high expression of MФs-feature genes and PBLs had high expression of platelet-feature genes, no matter how the onset conditions of BM and sepsis changed. In other words, it seemed that CSF cells always maintained a relatively high proportion of MФs and almost no platelets (no platelets were identified in CSF cells by SCRNA-SEQ) compared with PBLs in the development of sepsis-developed BM.

When simultaneously comparing the bulk transcriptomes between CSF cells in BM initial onset, unrefractory/refractory remission and recovery stages and PBLs in sepsis onset and recovered stages (CSF cells versus PBLs), it showed that the down-regulated DEGs with high $P$ values were almost highly expressed on MФs of CSF, while up-regulated DEGs with high $P$ values were almost highly expressed on platelets of PBLs (Fig. 9A). 
The expression of MФ-featured DEGs in CSF cells and PBLs was checked. The mRNA expression levels of TREM2 and SLCO2B1 in CSF cells and PBLs were measured through RT-qPCR, and it was found that CSF cells expressed significantly higher levels of TREM2 and SLCO2B1 than PBLs (Fig. 9B). The expression of VSIG4 in CSF cells and PBLs was measured through FCM-immunostaining, and the results (Fig. 9C) revealed that CSF-MN cells had a significantly higher proportion of the VSIG4 ${ }^{+}$cell subgroup than PBLMN cells. For SPP1, we measured the concentration of OPN in multiple CSF supernatants and blood sera acquired from septicemia-developed BM patients, and the results (Fig. 9D) showed that CSF supernatants contained significantly higher OPN levels than blood sera.

\section{Discussion}

The development of BM is a relatively complex process that brought certain difficulties to our research. First, BM could be induced by the infection of multiple pathogens, including both gram-positive and gramnegative bacteria. Second, BM was prone to occur in infants and young children, which resulted in individual differences in onset conditions due to their immature immune system. We believe our study is credible and representative, as it is based on systematic analysis of a large number of BM patients. The pathogens of BM patients in our study covered common species, and GBS, E. coli and S. pneumoniae infections accounted for most cases, which was in accordance with the BM incidence in other countries [39-41]. This meant that our study could be universally adopted by international researchers in further studies on BM.

At present, cellular studies on CSF cells have not received attention. One important reason is that CSF contains limited cell numbers. Some studies have attempted to characterize the phenotype of CSF cells by immunostaining combined with FCM [42-44]. However, as only a small set of surface markers were selected, the results are low resolution and may miss important cells. ScRNA-SEQ has been used in few studies to explore the heterogeneities of CSF cells $[26,45,46]$. These studies mainly focus on adult neurological diseases, which mean that ScRNA-SEQ implementation is easier to achieve because adults can provide a higher dose of CSF. In contrast, CSF could only be collected as much as $0.5 \mathrm{cc} \sim 1.0 \mathrm{cc}$ through routine lumbar puncture in our research, since BM patients are always at a younger age, and sampling too much CSF will endanger their health. Therefore, our research is the first trial dissecting the heterogeneities of CSF cells in acute CNS infection and childhood neurological diseases by scRNA-SEQ. Compared with other studies that only targeted the pathological condition in a specific period of disease progression, our study dynamically monitored the landscape of CSF cells with SCRNA-SEQ by targeting the whole period of BM development, which was more profound and comprehensive.

Our study first confirmed that CSF cells of BM patients with unsatisfactory therapeutic effects had different population structures and intercellular communications compared with those with satisfactory therapeutic effects, rather than just the extension of illness duration. More mDCs and pDCs especially appeared in the CSF during the refractory condition. This discovery provides new ideas for the treatment of refractory meningitis. In addition, we also identified two novel cell subtypes, cytokine ${ }^{+} \mathrm{NEU}$ s and cytokine ${ }^{+} \mathrm{MOs}$, which participated in neuroinflammation during CNS infection. The discovery of these 
two cell types explained why CSF had increased concentrations of cytokines in the process of BM. After all, the phenomenon that cytokine levels increase in CSF during BM has been reported [47]. More importantly, it clarified which BM stage led to increased cytokine levels in CSF, especially that cytokines would not increase in the refractory remission stages of BM, even if TCCs of CSF increased considerably.

In our study, we found that CSF always contained a higher proportion of MФs and almost no platelets than PBLs in sepsis-developed BM. The phenomenon that platelets did not appear in CSF may be because platelets lacked cellular structure, which resulted in platelets not penetrating through the BBB structure from blood into CSF. A study [45] found that CSF-MФ cells displayed microglia-like signatures, and they directly marked the CSF-MФ cell group as microglia-like cells. Therefore, the MФ difference between CSF and PBLs in BM progression might be because the MФs in CSF were microglia-derived cells rather than conventional MФs in the blood and could directly enter the CSF as residential CNS cells.

Our study found that the gene expression in CSF cells could reflect into the protein content of CSF supernatant, which meant that we could obtain the disease-related characteristics from the CSF supernatant based on the study of CSF cells. After all, compared with the direct study targeting CSF supernatant, the study on CSF cells was more likely to obtain valuable information since the relatively mature cytology research methods. Therefore, our study was like a bridge linking the biochemical indexes of CSF supernatant with the molecular expression of CSF cells. We hope that our research will not only help people understand the cellular immune process in CSF during BM but also provide positive references for studies on other neurological diseases.

\section{Conclusions}

In summary, our study displayed which types of immune cells enter CSF to participate in neuroimmunity against bacterial infection in BM and clarified their population structure and changing law in BM progression under clinical treatment by conducting in-depth dissections about the cell heterogeneities of CSF. The discovery of these two new cell subtypes, cytokine ${ }^{+}$NEUs and cytokine ${ }^{+}$MOs, explained why cytokine levels in CSF increased and which cell types played a pioneering role against bacterial invasion in BM. It also illustrated that the increased levels of cytokines in CSF mainly occurred in the initial onset stage of BM and would not increase in BM refractory stages even if TCCs of CSF increased considerably. The occurrence of refractory BM was not just an extension of onset duration but with changed heterogeneity and intercellular communication of CSF cells compared with unrefractory BM. Immune cells in CSF showed different characteristics compared with those in blood during sepsis-developed BM, since they expressed distinct molecular hallmarks and CSF cells contained a specific high proportion of MФs and almost no platelets. These findings not only provide novel insights for understanding the neuroinflammatory signatures of BM but also provide new therapeutic approaches in refractory BM.

\section{Abbreviations}

BM: Bacterial meningitis 
CNS: central nervous system

CSF: Cerebrospinal fluid

PBLs: Peripheral blood leukocytes

scRNA-SEQ: Single-cell RNA-sequencing

NEUs: Neutrophil cells

MOs: Monocyte cells

cMOs: conventional MOs

cNEUs: conventional NEUs

mDCs: Myeloid dendritic cells

mDC2s: Myeloid type- $₫$ dendritic cells

mDC1s: Myeloid type- $₫$ dendritic cells

NKs: Natural killer cells

pDCs: Plasmacytoid dendritic cells

MФs: Macrophages

PMNs: Polymorphonuclear cells

MNs: Mononuclear cells

bulkRNA-SEQ: Bulk RNA sequencing

ACK: Ammonium-chloride-potassium

DMEM: Dulbecco's modified eagle medium

FBS: Fetal bovine serum

PBS: Phosphate buffered solution

cDNA: Complementary DNA

UMls: Unique molecular identifiers

DEGs: Differentially expressed genes 
GO: Gene ontology

RT-qPCR: Reverse transcription and quantitative real-time polymerase chain reaction

$\mathrm{C}_{\mathrm{T}}$ : Cycle threshold

FCM: Flow cytometry

BSA: Bovine serum albumin

ELISA: Enzyme-linked immunosorbent assay

CXCL8: Interleukin-8

PEDF: Pigment epithelium-derived factor

OPN: Osteopontin

TCCs: Total cell counts

BP: Biological process

BBB: Blood brain barrier

\section{Declarations}

\section{Availability of data and materials}

Raw and processed data files for SCRNA-SEQ and bulkRNA-SEQ have been deposited under GEO accession numbers GSE163219, which includes three subseries: GSE163194, GSE163195 and GSE163196. Specifically, GSE163194 and GSE163195 were used for scRNA-SEQ and bulkRNA-SEQ of CSF cells, respectively, and GSE163196 was used for bulkRNA-SEQ of PBLs.

The other datasets used and/or analyzed during the current study are available from the corresponding author on reasonable request.

\section{Acknowledgements}

We are grateful to Junying Jia (Institute of Biophysics, Chinese Academy of Sciences, Beijing, China) and Deqin Feng (Institute of Microbiology, Chinese Academy of Sciences, Beijing, China) for help with singlecell sorting by FCM.

\section{Funding}


This work was supported by grants from Innovation Academy for Strategic Priority Research Program of Chinese Academy of Sciences (XDA17010503), the National Science and Technology Major Project (2018ZX10101004003003), and The Special Fund of the Pediatric Medical Coordinated Development Center (No. XTZD20180501), "Dengfeng" Talent Training Plan (DFL 20181201) and Beijing Excellent Talents Training Program (No. 2018000021469G274) of Beijing Hospitals Authority.

\section{Author Contributions}

Haihan Xiao and Haijuan Xiao designed and conducted the experiments; Yun Zhang discussed the draft paper; Lingyun Guo, Zhenzhen Dou, Linlin Liu, Liang Zhu, Wenya Feng, Bing Liu, Bing Hu and Tianming Chen aided for CSF and blood sampling; Gang Liu and Tingyi Wen conceived of and supervised the project, analyzed data, made figures, and wrote the paper; and all authors read and commented on the manuscript.

\section{Conflict of Interest}

The authors declare that they have no competing interests.

\section{Ethics approval and consent to participate}

This can be seen in the methods.

\section{Consent for publication}

Not applicable.

\section{References}

1. van de Beek D. Progress and challenges in bacterial meningitis. The Lancet 2012; 380(9854): 1623-4.

2. Baud O, Aujard Y. Chapter 114 - Neonatal bacterial meningitis. In: Dulac O, Lassonde M, Sarnat HB. Handbook of Clinical Neurology Vol. 112: Elsevier, 2013:1109-13.

3. Sadarangani M, Pollard AJ. Bacterial Meningitis in Childhood. In: Curtis N, Finn A, Pollard AJ. Hot Topics in Infection and Immunity in Children VIII. New York, NY: Springer New York, 2011:185-99.

4. Lucas MJ, Brouwer MC, van de Beek D. Neurological sequelae of bacterial meningitis. The Journal of infection 2016; 73(1): 18-27.

5. Barichello T, Fagundes GD, Generoso JS, Elias SG, Simoes LR, Teixeira AL. Pathophysiology of neonatal acute bacterial meningitis. Journal of medical microbiology 2013; 62(Pt 12): 1781-9. 
6. Ku LC, Boggess KA, Cohen-Wolkowiez M. Bacterial meningitis in infants. Clinics in perinatology 2015; 42(1): 29-45, vii-viii.

7. Kim KS. Acute bacterial meningitis in infants and children. Lancet Infect Dis 2010; 10(1): 32-42.

8. Davis LE. Acute Bacterial Meningitis. Continuum (Minneapolis, Minn) 2018; 24(5, Neuroinfectious Disease): $1264-83$.

9. Luo Y, Liao Z, Shu J, Zhang J, Yu X. Observation and analysis of the efficacy of dexamethasone in combination with anti-infectious treatment on the pediatric refractory purulent meningitis. Pakistan journal of pharmaceutical sciences 2020; 33(1(Special)): 489-94.

10. Peng HL, Hu Y, Chen HJ, Song PP, Jiang L. Risk factors for poor prognosis in children with refractory purulent meningitis and the discharge criteria. Journal of infection and public health 2018; 11(2): 238-42.

11. Sakka L, Coll G, Chazal J. Anatomy and physiology of cerebrospinal fluid. European annals of otorhinolaryngology, head and neck diseases 2011; 128(6): 309-16.

12. Hasbun R. Cerebrospinal fluid in central nervous system infections. 2014.

13. Agueda S, Campos T, Maia A. Prediction of bacterial meningitis based on cerebrospinal fluid pleocytosis in children. The Brazilian journal of infectious diseases : an official publication of the Brazilian Society of Infectious Diseases 2013; 17(4): 401-4.

14. Prajapati BS, Prajapati RB, Vora HD. Analysis of cerebrospinal fluid (CSF) in children. Pediatric Infectious Disease 2015; 7(1): 22-6.

15. Reshi Z, Nazir M, Wani W, Malik M, Iqbal J, Wajid S. Cerebrospinal fluid procalcitonin as a biomarker of bacterial meningitis in neonates. Journal of perinatology : official journal of the California Perinatal Association 2017; 37(8): 927-31.

16. Tan J, Kan J, Qiu G, et al. Clinical Prognosis in Neonatal Bacterial Meningitis: The Role of Cerebrospinal Fluid Protein. PloS one 2015; 10(10): e0141620.

17. Srinivasan L, Kilpatrick L, Shah SS, Abbasi S, Harris MC. Cerebrospinal fluid cytokines in the diagnosis of bacterial meningitis in infants. Pediatric research 2016; 80(4): 566-72.

18. Andrews TS, Hemberg M. Identifying cell populations with scRNASeq. Molecular aspects of medicine 2018; 59: 114-22.

19. Guo LY, Zhang ZX, Wang X, et al. Clinical and pathogenic analysis of 507 children with bacterial meningitis in Beijing, 2010-2014. International journal of infectious diseases : IJID : official publication of the International Society for Infectious Diseases 2016; 50: 38-43.

20. Sasagawa Y, Danno H, Takada H, et al. Quartz-Seq2: a high-throughput single-cell RNA-sequencing method that effectively uses limited sequence reads. Genome biology 2018; 19(1): 29.

21. Chen S, Zhou Y, Chen Y, Gu J. fastp: an ultra-fast all-in-one FASTQ preprocessor. Bioinformatics 2018; 34(17): i884-i90.

22. Korsunsky I, Millard N, Fan J, et al. Fast, sensitive and accurate integration of single-cell data with Harmony. Nature methods 2019; 16(12): 1289-96. 
23. Chen J, Tan $Y$, Sun F, et al. Single-cell transcriptome and antigen-immunoglobin analysis reveals the diversity of B cells in non-small cell lung cancer. Genome biology 2020; 21(1): 152.

24. Chen YP, Yin JH, Li WF, et al. Single-cell transcriptomics reveals regulators underlying immune cell diversity and immune subtypes associated with prognosis in nasopharyngeal carcinoma. Cell research 2020; 30(11): 1024-42.

25. Mulder K, Patel AA, Kong WT, et al. Cross-tissue single-cell landscape of human monocytes and macrophages in health and disease. Immunity 2021; 54(8): 1883-900 e5.

26. Schafflick D, Xu CA, Hartlehnert M, et al. Integrated single cell analysis of blood and cerebrospinal fluid leukocytes in multiple sclerosis. Nature communications 2020; 11(1): 247.

27. Szabo PA, Levitin HM, Miron M, et al. Single-cell transcriptomics of human T cells reveals tissue and activation signatures in health and disease. Nature communications 2019; 10(1): 4706.

28. Wu X, Liu Y, Jin S, et al. Single-cell sequencing of immune cells from anticitrullinated peptide antibody positive and negative rheumatoid arthritis. Nature communications 2021; 12(1): 4977.

29. Xue D, Tabib T, Morse C, Lafyatis R. Transcriptome landscape of myeloid cells in human skin reveals diversity, rare populations and putative DC progenitors. Journal of dermatological science 2020; 97(1): 41-9.

30. Zheng Y, Chen Z, Han Y, et al. Immune suppressive landscape in the human esophageal squamous cell carcinoma microenvironment. Nature communications 2020; 11(1): 6268.

31. Rojas-Rivera D, Hetz C. TMBIM protein family: ancestral regulators of cell death. Oncogene 2015; 34(3): 269-80.

32. Zechendorf E, O'Riordan CE, Stiehler L, et al. Ribonuclease 1 attenuates septic cardiomyopathy and cardiac apoptosis in a murine model of polymicrobial sepsis. JCl insight 2020; 5(8).

33. Wang W, Zheng $Y$, Sun S, et al. A genome-wide CRISPR-based screen identifies $<i>K A T 7</ i>$ as a driver of cellular senescence. Science translational medicine 2021; 13(575): eabd2655.

34. Villar J, Segura E. Decoding the Heterogeneity of Human Dendritic Cell Subsets. Trends in immunology 2020; 41(12): 1062-71.

35. Cao L, Prithviraj P, Shrestha R, et al. Prognostic Role of Immune Checkpoint Regulators in Cholangiocarcinoma: A Pilot Study. Journal of clinical medicine 2021; 10(10): 2191.

36. Nakamura K, Smyth MJ. Myeloid immunosuppression and immune checkpoints in the tumor microenvironment. Cellular \& molecular immunology 2020; 17(1): 1-12.

37. Sochocka M, Diniz BS, Leszek J. Inflammatory Response in the CNS: Friend or Foe? Molecular neurobiology 2017; 54(10): 8071-89.

38. Klein RS, Hunter CA. Protective and Pathological Immunity during Central Nervous System Infections. Immunity 2017; 46(6): 891-909.

39. Okike IO, Johnson AP, Henderson KL, et al. Incidence, etiology, and outcome of bacterial meningitis in infants aged $<90$ days in the United kingdom and Republic of Ireland: prospective, enhanced, 
national population-based surveillance. Clinical infectious diseases : an official publication of the Infectious Diseases Society of America 2014; 59(10): e150-7.

40. Ouchenir L, Renaud C, Khan S, et al. The Epidemiology, Management, and Outcomes of Bacterial Meningitis in Infants. Pediatrics 2017; 140(1).

41. Agrawal S, Nadel S. Acute bacterial meningitis in infants and children: epidemiology and management. Paediatric drugs 2011; 13(6): 385-400.

42. Amundson B, Lai L, Mulligan MJ, et al. Distinct cellular immune properties in cerebrospinal fluid are associated with cognition in HIV-infected individuals initiating antiretroviral therapy. Journal of neuroimmunology 2020; 344: 577246.

43. Lueg G, Gross CC, Lohmann $\mathrm{H}$, et al. Clinical relevance of specific T-cell activation in the blood and cerebrospinal fluid of patients with mild Alzheimer's disease. Neurobiology of aging 2015; 36(1): 819.

44. Maxeiner HG, Rojewski MT, Schmitt A, Tumani H, Bechter K, Schmitt M. Flow cytometric analysis of T cell subsets in paired samples of cerebrospinal fluid and peripheral blood from patients with neurological and psychiatric disorders. Brain, behavior, and immunity 2009; 23(1): 134-42.

45. Farhadian S, Mehta S, Zografou C, et al. Single cell RNA sequencing reveals a novel, microglia-like cell type in cerebrospinal fluid during virologically suppressed HIV. 2018.

46. Pappalardo J, Zhang L, Pecsok M, et al. Transcriptomic and clonal characterization of $T$ cells in the human central nervous system. Science immunology 2020; 5.

47. Prasad R, Kapoor R, Srivastava R, Mishra OP, Singh TB. Cerebrospinal fluid TNF-alpha, IL-6, and IL-8 in children with bacterial meningitis. Pediatric neurology 2014; 50(1): 60-5.

\section{Figures}


A

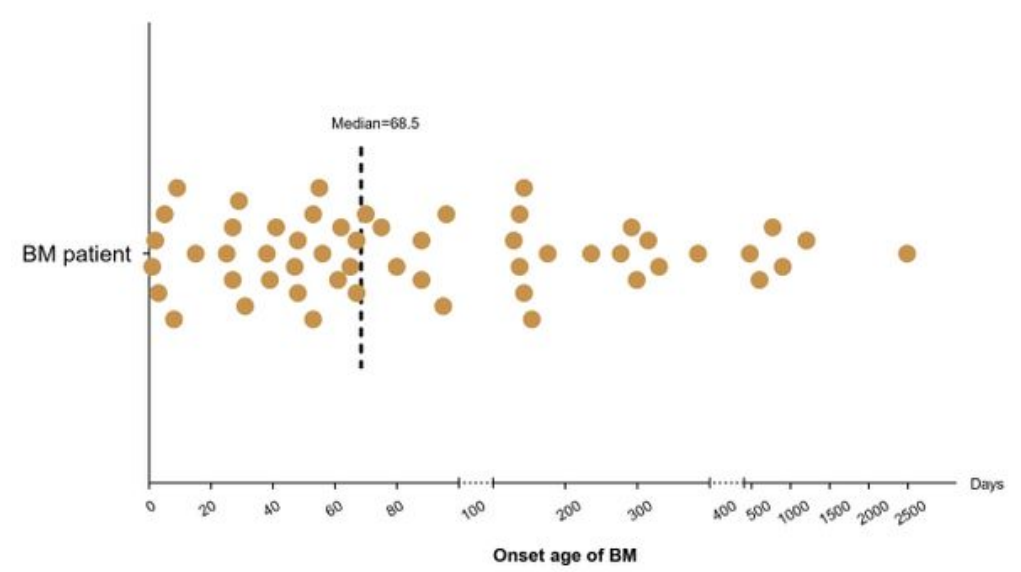

B

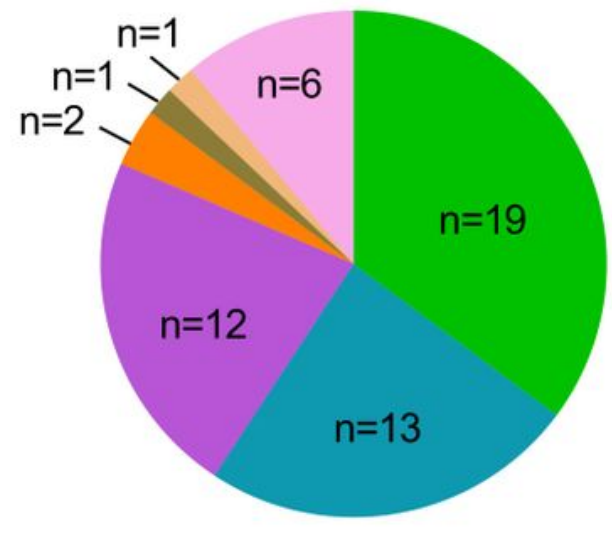

$$
\begin{aligned}
& \text { BM pathogens } \\
& \text { Streptococcus agalactiae } \\
& \text { Streptococcus pneumoniae } \\
& \text { Escherichia coli } \\
& \text { Listeria monocytogenes } \\
& \text { Enterococcus Faecium } \\
& \text { Neisseria meningitidis } \\
& \text { Unclear pathogen }
\end{aligned}
$$

BM patients

C

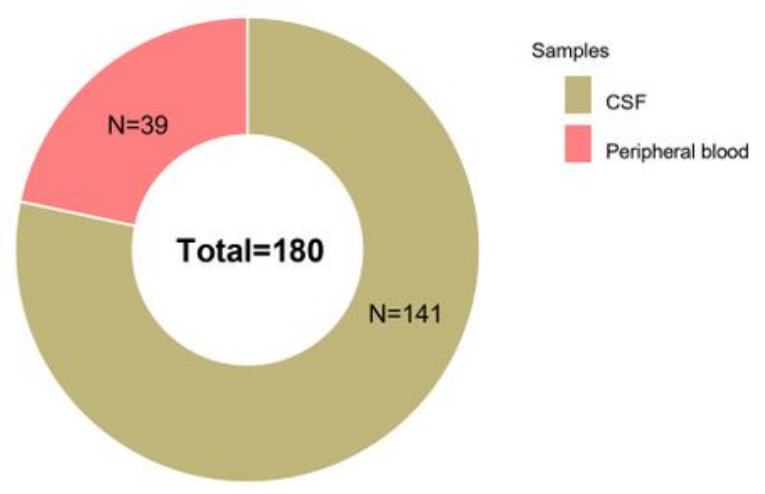

Collected samples from BM patients

\section{Figure 1}

Characteristics of the study cohort. (A) Scatter plot shows the onset ages of BM patients collected in the study. (B) Pie chart shows the pathogens of BM patients and cases in each of them. (C) Pie chart showed the sample numbers of CSF and peripheral blood collected from BM patients. 
A

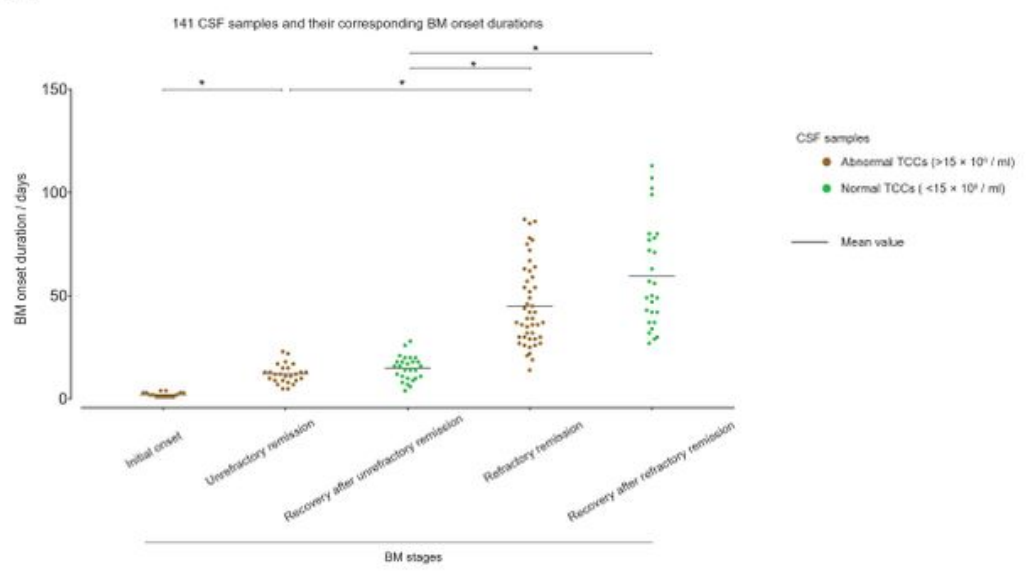

B

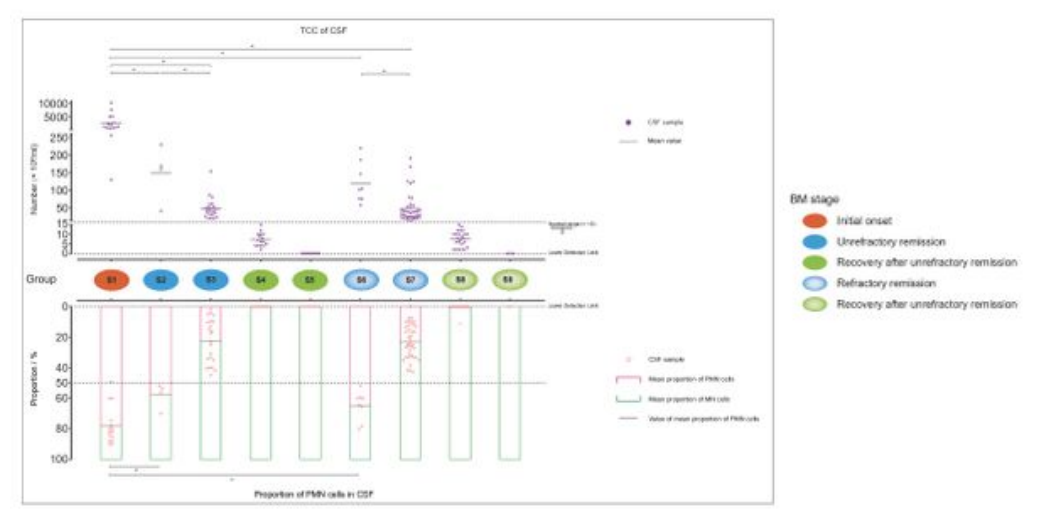

C

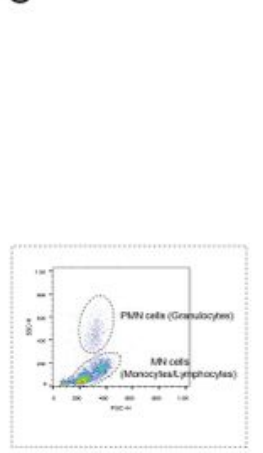

PBLs (referenco)

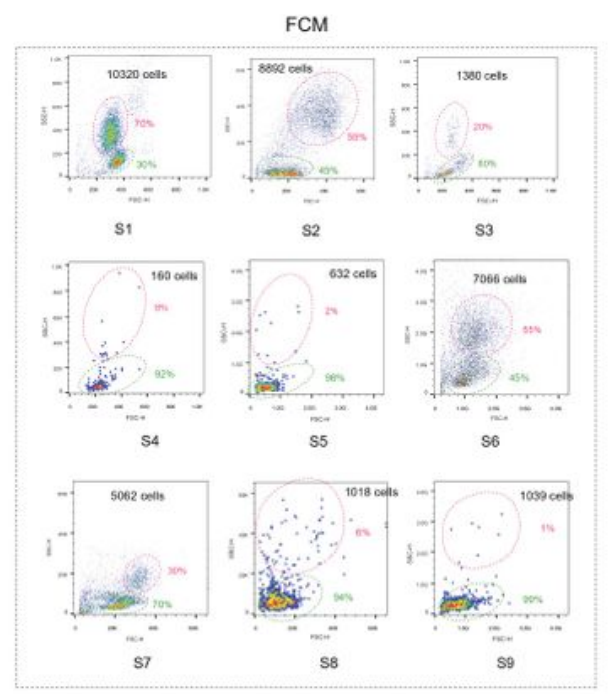

PMN-cells

MN-cells

CSF cells in different BM stages

\section{Figure 2}

The relationship between CSF cell characteristics and BM onset conditions. (A) Dot plot shows the CSF samples and the five BM onset stages they represent: initial onset, unrefractory remission, recovery following unrefractory remission, refractory remission and recovery following refractory remission, according to CSF cells and onset durations. (B) Bar charts show the characteristics of TCCs (upper), PMNs and MNs (lower) in CSF in different BM onset stages, which are classified into 9 groups (S1 S9). 
(C) Pseudocolor images show representative performances of CSF cells in different BM stages under FCM. PBLs were taken as a reference. PMN cells and MN cells in CSF cells and PBLs are marked with dashed pink and green circles respectively.

A
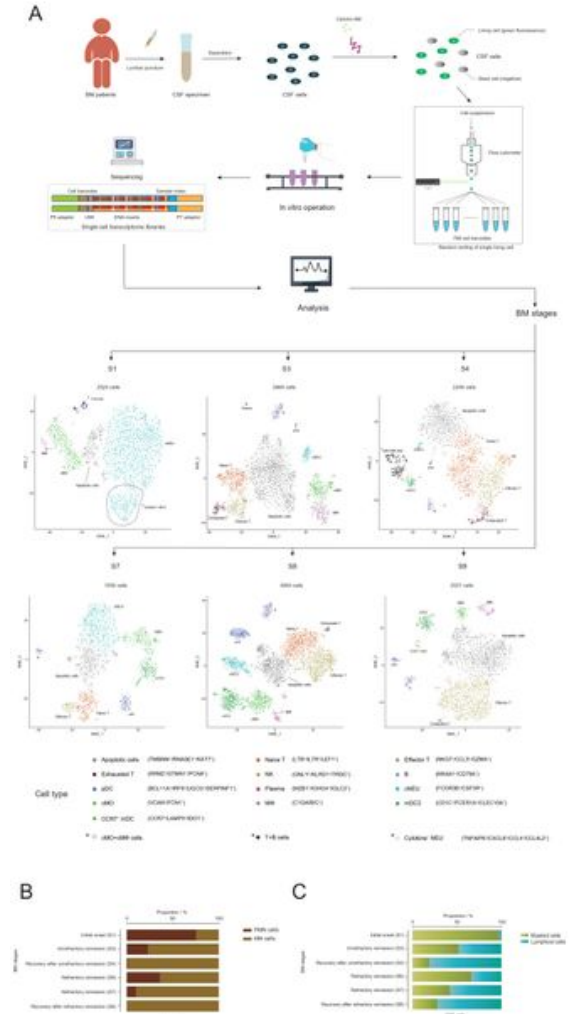

D

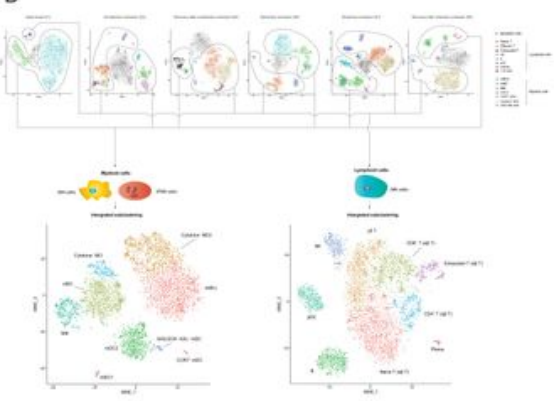

$E$

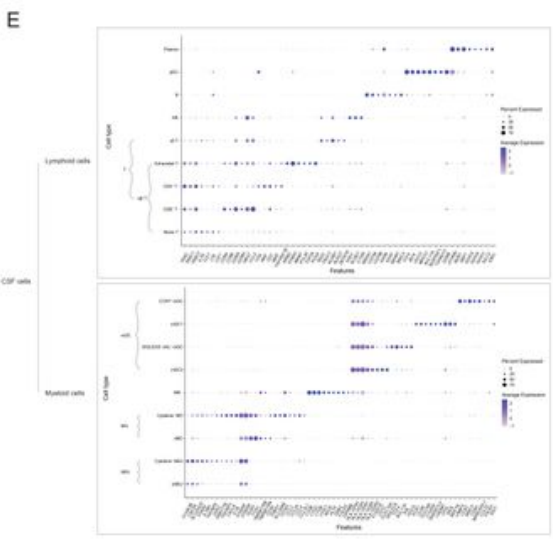

Figure 3 
scRNA-SEQ reveals the cell heterogeneities of CSF in BM progression. (A) SCRNA-SEQ workflow and t-SNE plots show the cell heterogeneities of CSF in the initial onset, unrefractory remission, recovery following unrefractory remission, refractory remission and recovery following refractory remission stages of BM. (B) Bar chart shows the proportions of PMN and MN cells in CSF in different BM stages, which were identified by SCRNA-SEQ. (C) Bar chart shows the proportions of myeloid and lymphoid cells in CSF in different BM stages, which were identified by scRNA-SEQ. (D) t-SNE plots show the immune cell subtypes of CSF in BM, which are achieved through integrative clustering of myeloid or lymphoid cells in each BM stage. (E) Bubble plots show the feature genes of cell subtypes in CSF. Shades of blue represent the relative abundance (the higher the abundance, the bluer the color), and bubble sizes represent the expression level (the higher the expression, the larger the size) of each gene. Cell subtypes are displayed by myeloid and lymphoid cells. 

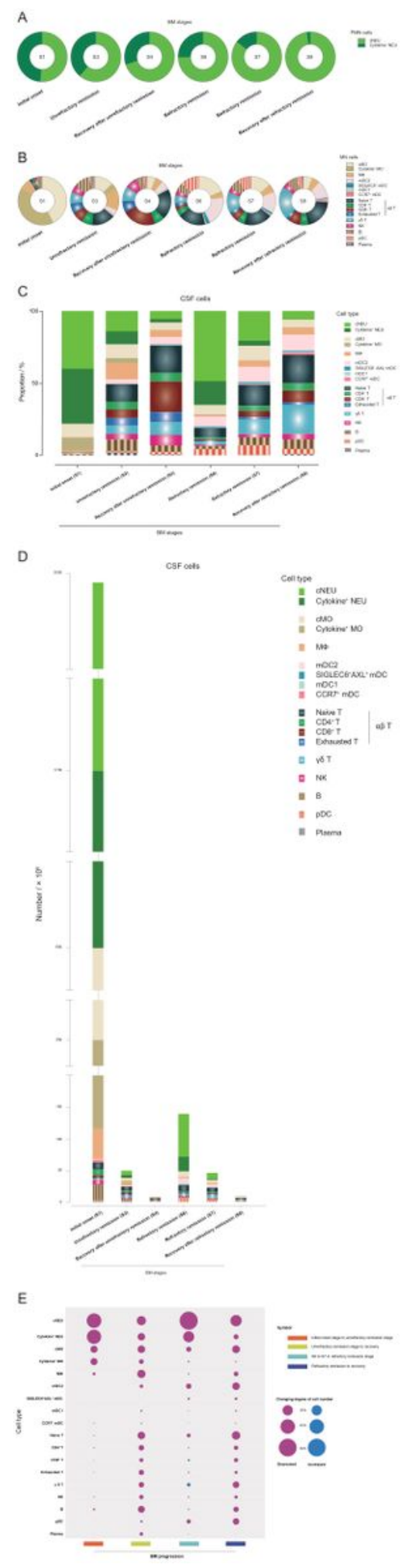

\section{Figure 4}

Population structure of CSF cells in BM progression. (A-B) Pie charts show the population structures of the PMN cell group (A) and MN cell group (B) of CSF in BM initial onset, unrefractory remission, recovery after unrefractory remission, refractory remission and recovery after refractory remission stages. (C-D) Bar charts show the population structures of CSF cells (C) and quantities of CSF cell types (D) in BM initial onset, unrefractory remission, recovery after unrefractory remission, refractory remission and 
recovery after refractory remission stages. (E) Bubble plot shows the changing degrees in the number of CSF cell subtypes during the process of BM progression from initial onset to unrefractory remission stage, unrefractory remission stage to recovery, S6 to S7 in refractory remission stage and refractory remission stage to recovery. The higher the changing degree is, the larger the size. Red and blue bubbles represent decreased and increased changing numbers, respectively.

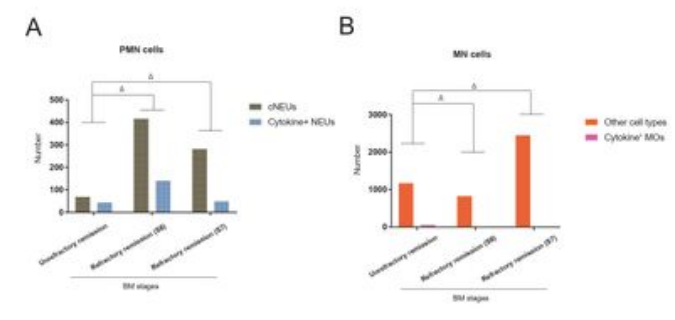

C
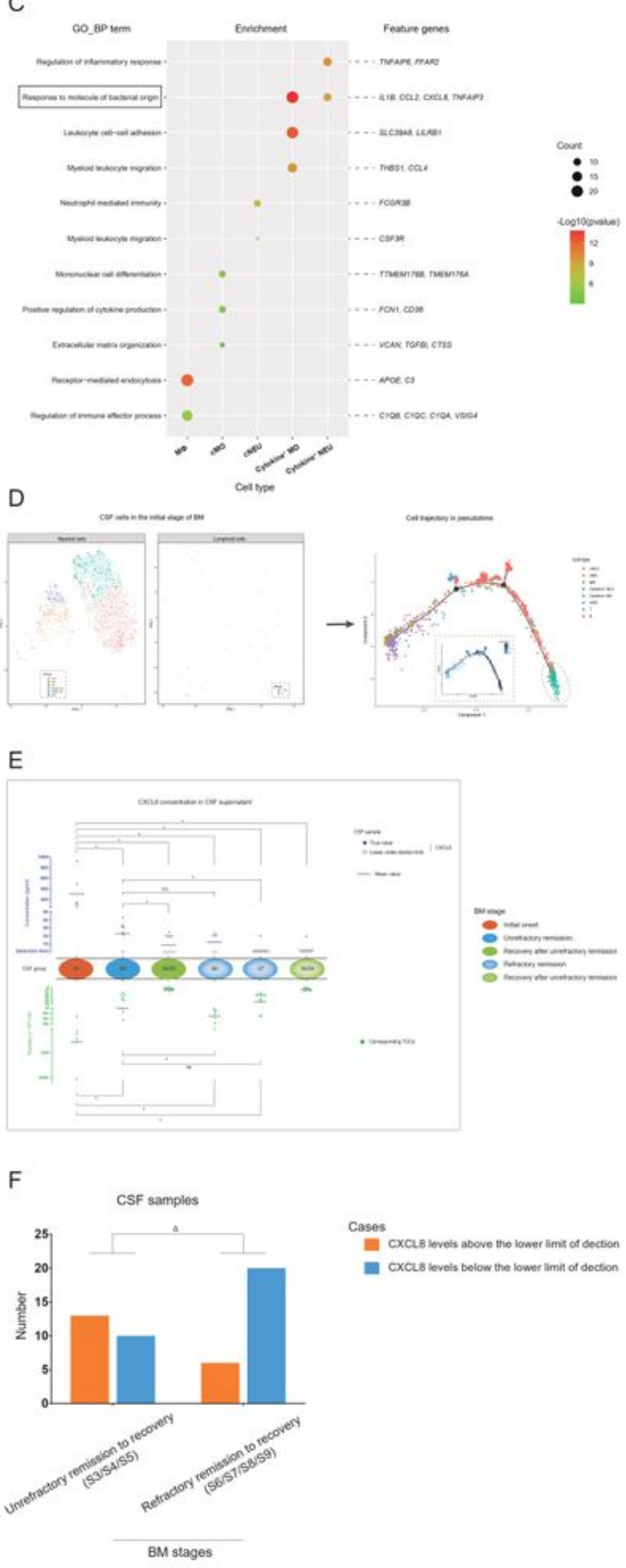

Figure 5 
Immune roles of cytokine ${ }^{+}$NEUs and cytokine ${ }^{+}$MOs in BM progression. (A-B) Bar charts show the numbers of cytokine ${ }^{+}$NEUs in PMN cells (A) and cytokine ${ }^{+}$MOs in MN cells (B) detected by scRNA-SEQ at BM unrefractory and refractory remission stages. (C) Bubble plot shows the GO-BP enrichments targeting the feature genes of MФs, cMOs, cNEUs, cytokine ${ }^{+}$NEUs and cytokine ${ }^{+} \mathrm{MO}$ s of CSF. Green to red indicate the logarithmic $P$ value of each GO-BP term from low to high (the more significant the $P$ value, the redder the color) (scaled). Bubble sizes indicate the number of genes enriched for each GO-BP term. (D) Cell trajectory plot shows the pseudotime analysis of MФs, cMOs, cNEUs, cytokine ${ }^{+}$NEUs, cytokine ${ }^{+}$MOs, mDCs, T cells and B cells of CSF in the initial onset stage of BM. The cytokine ${ }^{+}$NEUs are emphasized with dashed circles. (E) Dot plots show the CXCL8 levels in supernatant (upper) and TCCs (lower) of CSF samples in BM initial onset, unrefractory remission, recovery following unrefractory remission, refractory remission and recovery following refractory remission stages. CXCL8 levels above or below the lower detection limit are marked by solid blue points or blue circles, respectively. (F) Bar chart shows the numbers of CSF samples in which the CXCL8 levels are above or below the lower detection limit in the BM unrefractory-remission/recovery and refractory-remission/recovery stages. *: t-test, $\mathrm{p}<$ 0.05. $\Delta$ : Chi-square test, $\mathrm{p}<0.05$. 

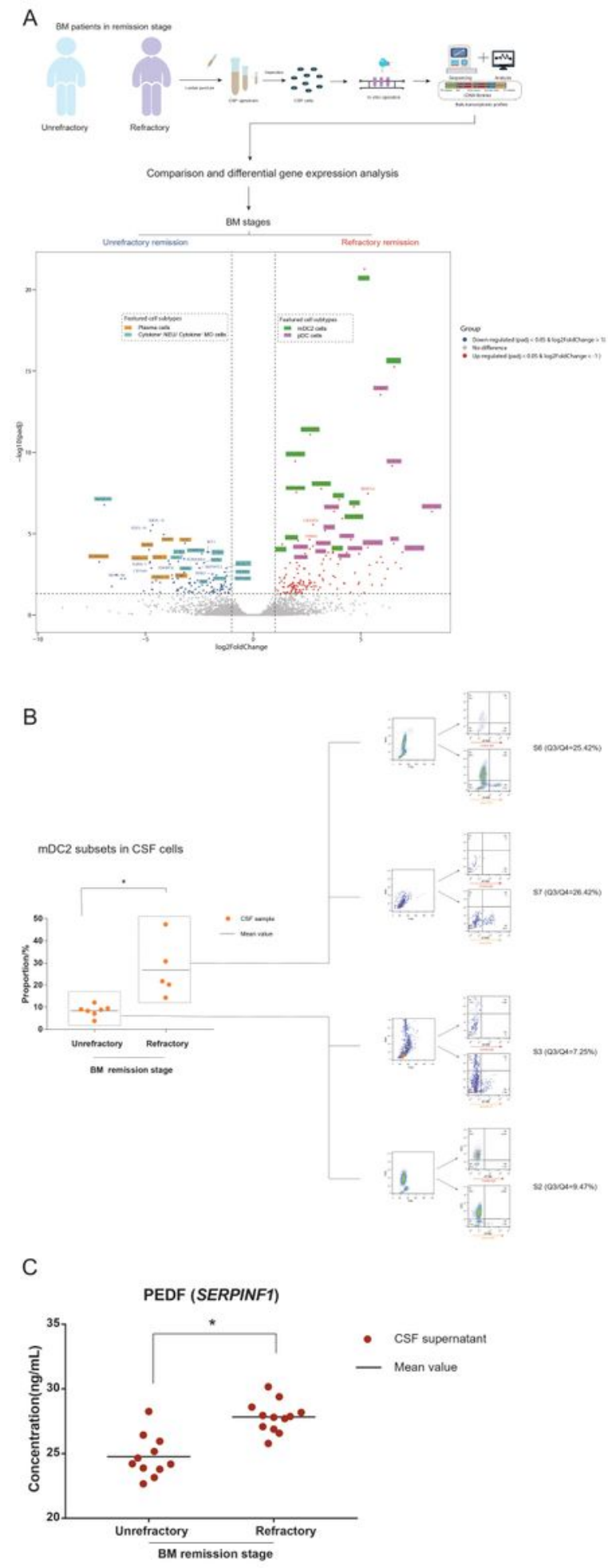

\section{Figure 6}

CSF cells in the BM refractory remission stage feature higher proportions of $\mathrm{mDC} 2 \mathrm{~s}$ and $\mathrm{pDCs}$ than those in the BM unrefractory remission stage. (A) BulkRNA-SEQ workflow and volcano plot show the DEGs identified by bulk transcriptomic comparison between CSF cells in the BM unrefractory and refractory remission stages. In the volcano plot, the $\mathrm{x}$-axis shows log twofold change (log2FC) values, and the $y$-axis shows negative log ten padj values. Each gene is represented by a single spot. Significantly down- 
regulated ( $\log 2 \mathrm{FC} \leq-2 \&$ padj $\leq 0.01)$ and up-regulated (log2FC $\geq 2 \&$ padj $\leq 0.01)$ DEGs are represented by blue and red spots, respectively. The thresholds of log2FC and padj are plotted with dashed lines. The names of down-regulated and up-regulated genes with top padj values are listed next to the corresponding spots. The background colors of the gene names represent the cell type of CSF that highly expresses the corresponding gene. (B) Dot plot and pseudocolor images show the proportions of $\mathrm{mDC} 2$ cells (CD1C $\mathrm{C}^{+}$subgroup) in CSF cells at BM unrefractory and refractory remission stages. (C) Dot plot shows the PEDF (encoded by SERPINF1) levels in supernatants of CSF samples at BM unrefractory and refractory remission stages. $*$ : $t$ test, $p<0.05$. 

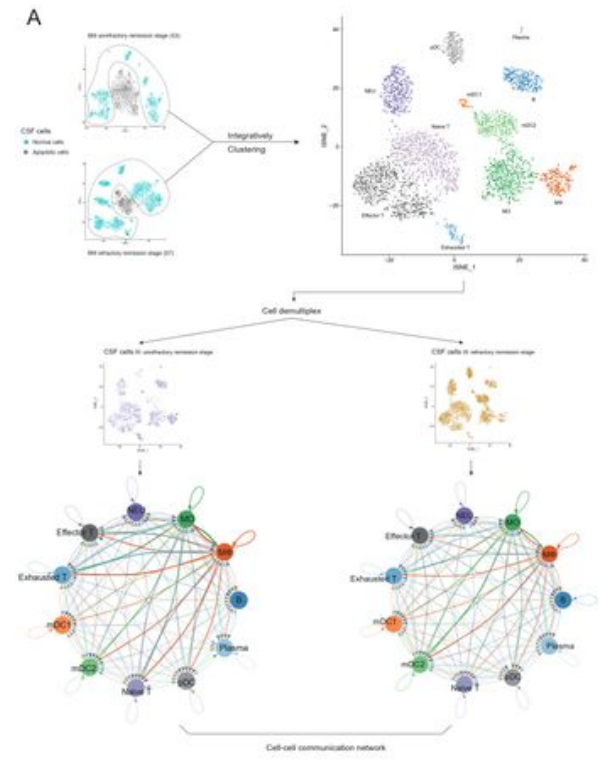

。

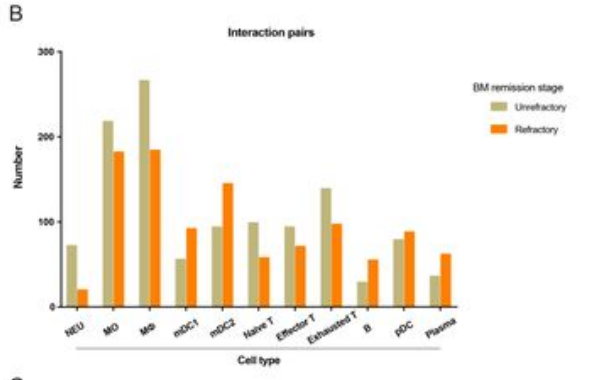

C

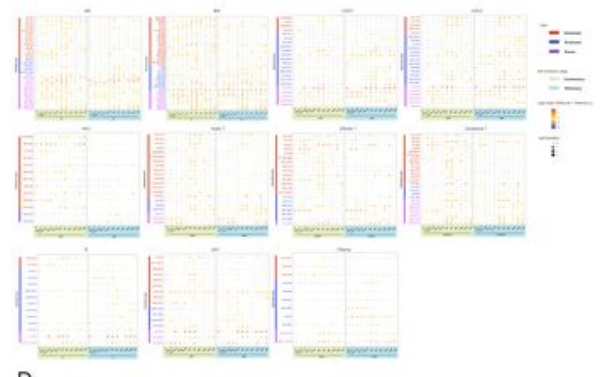

D
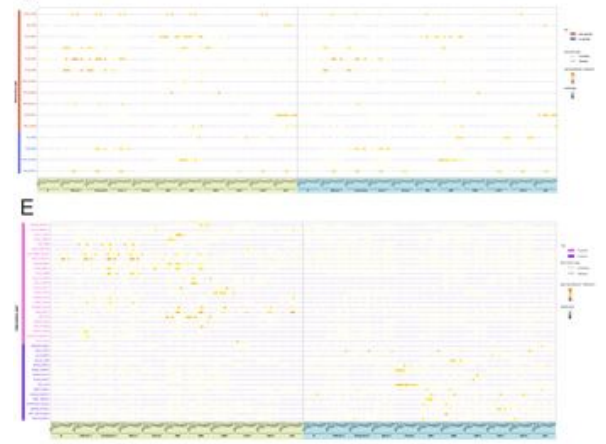

Figure 7

The different intercellular communications between CSF cells in the BM unrefractory and refractory remission stages. (A) t-SNE plots show the workflow for the analysis of intercellular communications, and network diagrams show the cell-cell interactions between each cell type of CSF in the unrefractory and refractory remission stages. In the network diagrams, the line color is the same as the cell type that expresses the ligands. The lines connect to the cell types that express the cognate receptors. The line 
thickness is proportional to the number of ligands when cognate receptors are present in the recipient cell type. The loops indicate autocrine circuits. (B) Bar chart shows the number of ligand-receptor interactions of each CSF cell type in the unrefractory and refractory remission stages. (C) Bubble plots show the ligand-receptor interactions with changed levels between the unrefractory and refractory remission stages of MOs, MФs, mDC1s, mDC2s, NEUs, naïve T cells, effector T cells, exhausted T cells, $B$ cells, pDCs and plasma cells. Types of changed levels are marked with multiple colors: weakened (red), enhanced (blue) and roved (purple). (D) Bubble plot shows down-regulated and up-regulated interactions among all CSF cell types between the unrefractory and refractory remission stages. (E) Bubble plot shows turned-off and turned-on interactions of CSF cells in the unrefractory and refractory remission stages. In these bubble plots, bubble colors indicate the means of the average expression levels of interacting molecule 1 in cluster 1 and interacting molecule 2 in cluster 2 . Bubble sizes indicate $P$ values. The stronger the interaction is, the redder the color, and the larger the size. 
A

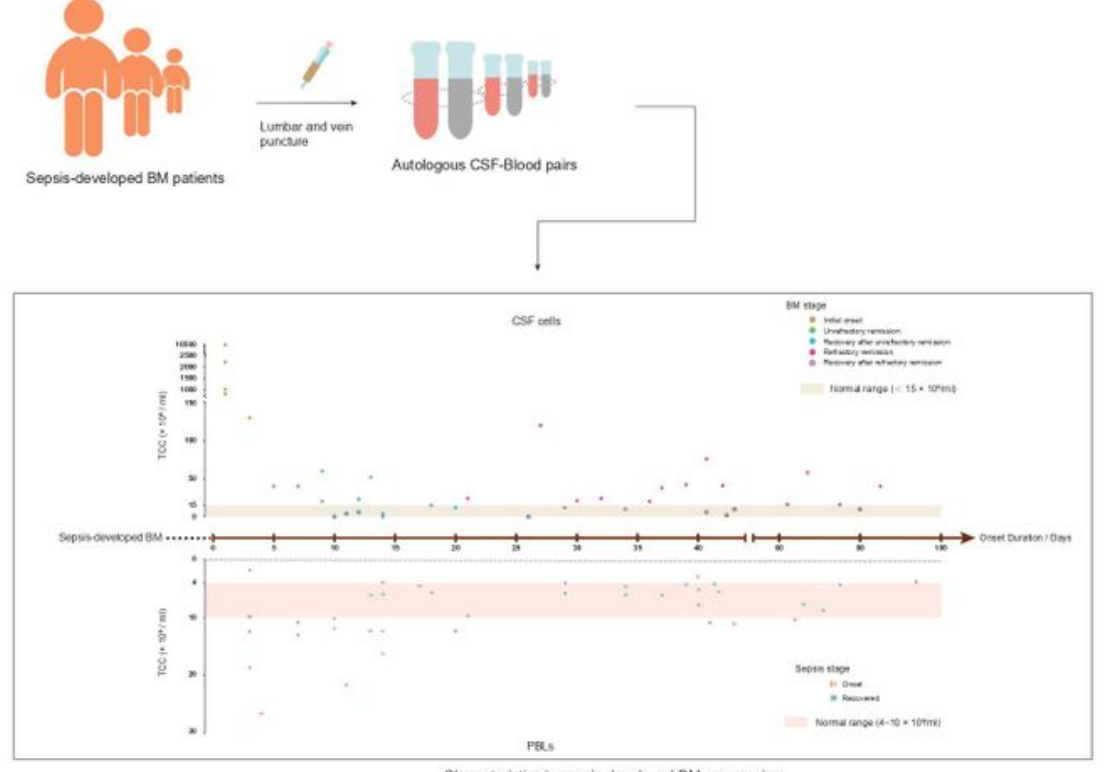

Characteristics in sepsis-developed BM progression

B
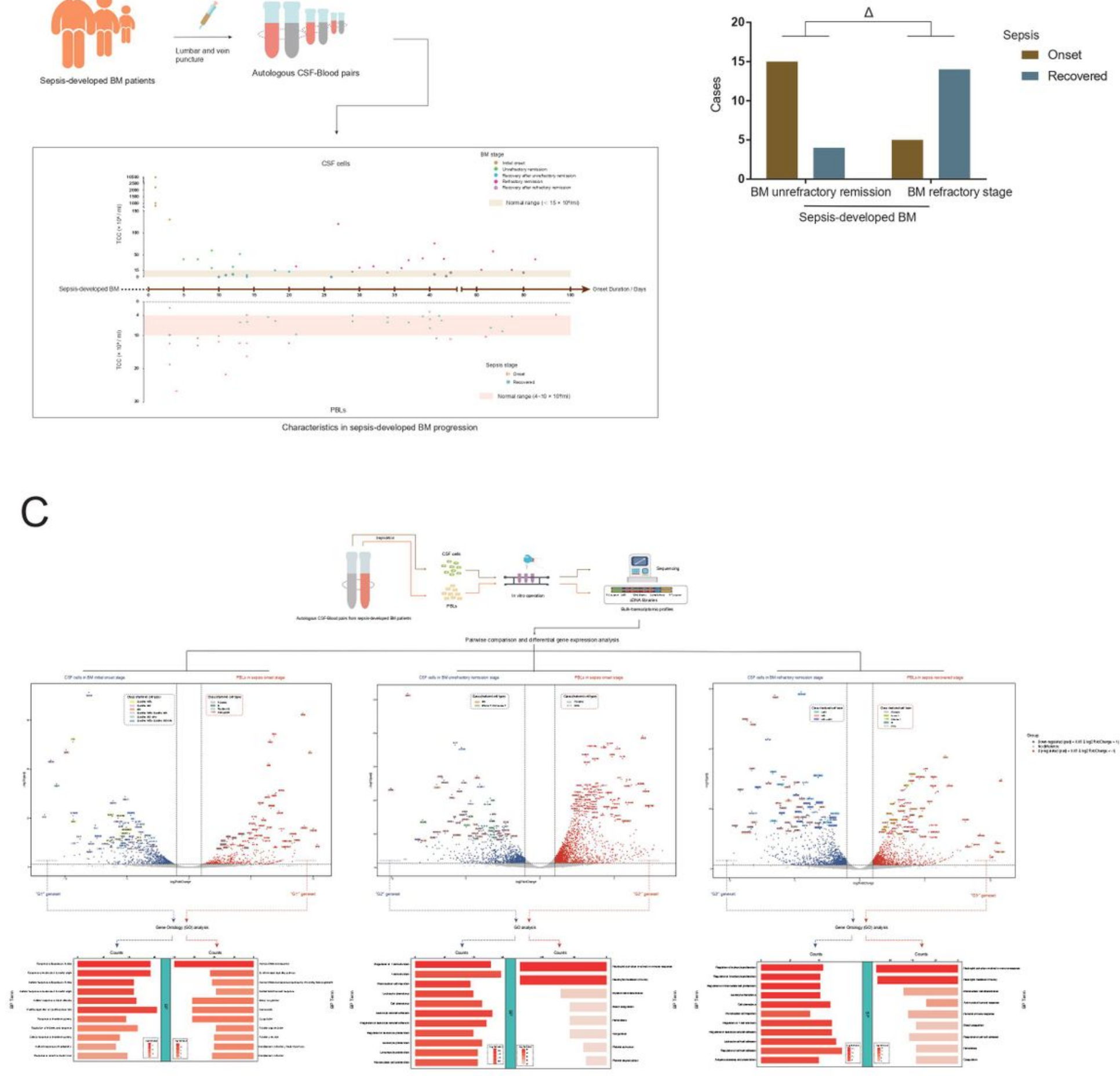

Figure 8

Different molecular hallmarks between CSF cells and PBLs in sepsis-developed BM. (A) Dot plots show the characteristics of CSF cells and PBLs and their represented BM and sepsis onset stages in sepsisdeveloped BM. (B) Bar chart shows the numbers of PBL samples in sepsis onset or recovered stage during the periods of $\mathrm{BM}$ initial onset to recovery after unrefractory remission and refractory remission to recovery. (C) BulkRNA-SEQ workflow, and volcano plots show the DEGs of pairwise bulk transcriptomic 
comparison between CSF cells in BM initial onset stage and PBLs in sepsis onset stage, CSF cells in BM unrefractory remission stage and PBLs in sepsis onset stage, and CSF cells in BM refractory remission stage and PBLs in sepsis recovered stage, and bar charts show the terms of GO-BP enrichments targeting the down-regulated and up-regulated DEGs in the three groups of comparison. In the volcano plots, the $x$ axis shows log2FC values, and the $y$-axis shows negative log ten padj values. Each gene is represented by a single spot. Significantly down-regulated $(\log 2 \mathrm{FC} \leq-2 \& \operatorname{padj} \leq 0.01)$ and up-regulated $(\log 2 \mathrm{FC} \geq 2$ $\&$ padj $\leq 0.01)$ genes are represented by blue and red spots, respectively. The thresholds of log2FC and padj are plotted with dashed lines. The names of down-regulated and up-regulated genes with top padj values are listed next to the corresponding spots. The background colors of down-regulated and upregulated gene names represent the cell types of CSF and PBLs, respectively, which highly express the corresponding gene. In the bar charts, shades of red indicate the logarithmic $P$ value of each GO-BP term from low to high (the more significant the $P$ value is, the redder the color) (scaled). Bar lengths indicate the number of genes enriched for each GO-BP term. $\Delta$ : Chi-square test, $p<0.05$. 

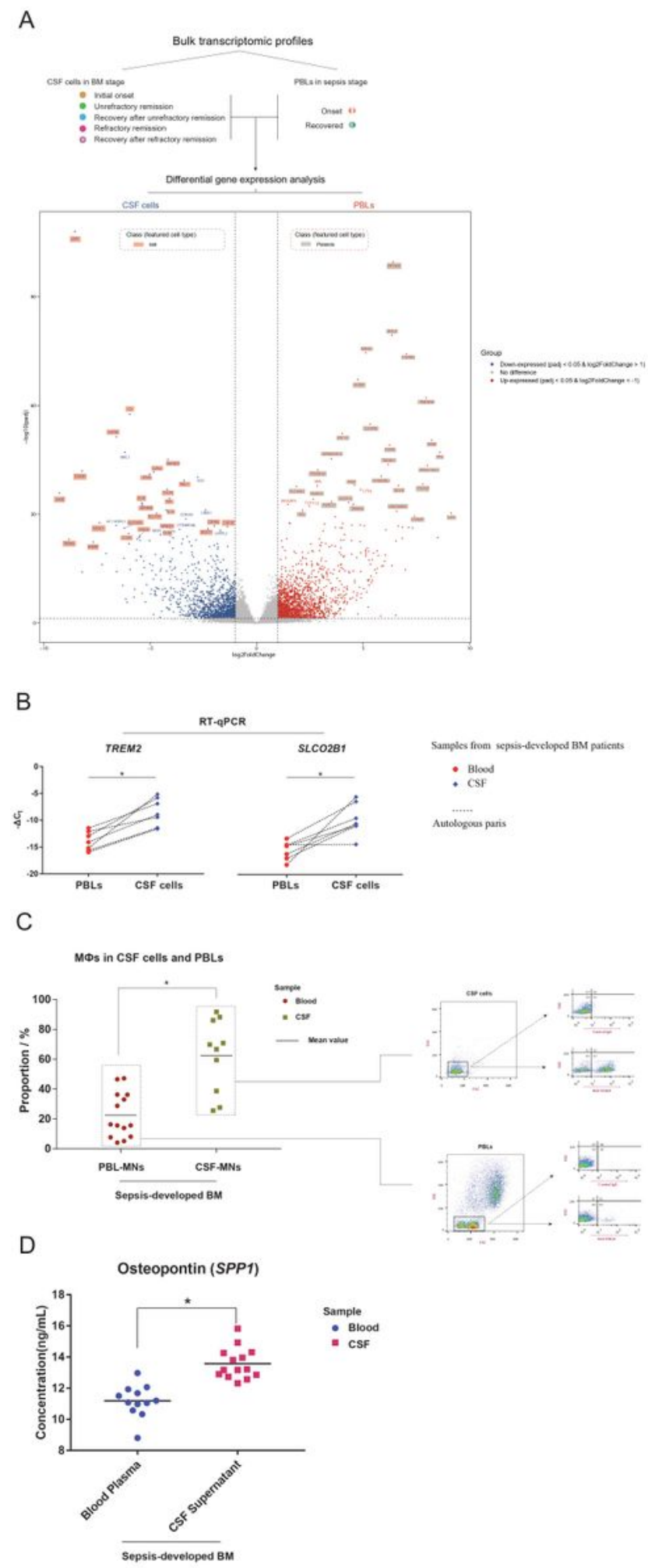

\section{Figure 9}

CSF cells always contain a higher proportion of MФs and almost no platelets than PBLs in sepsisdeveloped BM. (A) Volcano plot show the DEGs of the simultaneous bulk transcriptome comparison between CSF cells and PBLs in different onset stages of sepsis-developed BM. The x-axis shows log2FC values, and the $y$-axis shows negative log ten padj values. Each gene is represented by a single spot. Significantly down-regulated (log2FC $\leq-2 \&$ padj $\leq 0.01)$ and up-regulated $(\log 2 \mathrm{FC} \geq 2 \& \operatorname{padj} \leq 0.01)$ 
genes are represented by blue and red spots, respectively. The thresholds of log2FC and padj are plotted with dashed lines. The names of down-regulated and up-regulated genes with top padj values are listed next to the corresponding spots. The background colors of down-regulated and up-regulated gene names represent the cell types of CSF and PBLs, respectively, which highly express the corresponding gene. (B) Bar charts show the relative mRNA expression of TREM2 (left) and SLCO2B1 (right) in autologous CSF cells and PBLs by RT-qPCR. (C) Dot plot and pseudocolor images show the proportions of MФs (VSIG4 ${ }^{+}$ subgroup) in CSF-MN and PBL-MN cells. (D) Dot plot showe the OPN (encoded by SPP1) levels in CSF supernatants and blood sera by ELISA. *: t test, $p<0.05$.

\section{Supplementary Files}

This is a list of supplementary files associated with this preprint. Click to download.

- SupplementaryMaterials.pdf

- Table.S1.xIsx

- Table.S2.xlsx

- Table.S3.xlsx

- Table.S4.pdf 